\title{
Twitter under Storm: Social Media Response Sensitization in the Caribbean
}

\author{
Danilo Trinidad Pérez-Rivera ${ }^{1}$, Alexis Josué Martínez-Martínez ${ }^{2}$, Adriana del Mar \\ Pons-Calvo ${ }^{3}$, Alejandro Rodríguez Natal ${ }^{2}$, Sergio Andrés Dávila-Santana ${ }^{3}$
}

${ }^{1}$ Center for Neural Science, New York University, New York, NY 10003

${ }^{2}$ Mayagüez Campus, University of Puerto Rico, Mayagüez, PR 00682

${ }^{3}$ Medical Sciences Campus, University of Puerto Rico, San Juan, PR 00921

\begin{abstract}
Climate change has led to rising sea levels and warmer sea surface temperatures. These factors contribute greatly to the intensity of hurricanes and floods they provoke. Projections estimate there will be an increase of $45 \%$ to $87 \%$ in the frequency of Category $>4$ hurricanes originating in the Atlantic Basin, which typically impact the Caribbean and Continental United States of America. During the 2019 Hurricane Season, there were 20 depressions, 18 storms, 6 hurricanes and 3 major hurricanes. Through this work, we explored the response on Social Media to these natural phenomena as a function of their trajectory, intensity, and previous exposure of the population to intense natural disasters. Data was collected through the Twitter API. The influences of hurricane proximity and intensity on volume of Social Media production was explored. Hurricane Dorian, with its trajectory strongly threatening the previously exposed Puerto Rico, and eventually causing widespread damage in the Abaco Islands of the Bahamas, presented the strongest case for the evaluation of the dichotomy of responses between populations with differences in previous history of exposure. The landscape of historic hurricane exposure Caribbean has radically changed in recent years. Taking advantage of Big Data to help elucidate these dynamics could be instrumental in the tailoring of emergency preparedness plans and the effective design of mental health first aid strategies.
\end{abstract}

Keywords: Social Network Analysis, Computational Linguistics, Disaster Psychology

\section{Resumen}

El cambio climático ha provocado un aumento en el nivel del mar y temperaturas más cálidas en su superficie. Proyecciones estiman que factores contribuirán a un aumento de $45 \%$ a $87 \%$ en la frecuencia de los huracanes de categoría 4 y 5 que se originan en la cuenca del Atlántico. Durante la temporada de huracanes de 2019, hubo 20 depresiones, 18 tormentas, 6 huracanes y 3 huracanes de alta categoría. A través de este trabajo, investigamos la respuesta en las redes sociales a estos fenómenos naturales en función de su trayectoria, intensidad y exposición previa de la población a desastres naturales intensos. Los datos se recopilaron a través de la API (Interfaces de programación de aplicaciones) de Twitter. Se exploró cómo influye la proximidad y la intensidad de la tormenta tropical de interés, en el volumen del contenido generado en las redes sociales. El huracán Dorian amenazó fuertemente a Puerto Rico y eventualmente causó daños generalizados en las Islas Ábaco de las Bahamas. Esta situación sienta las bases para una evaluación de la dicotomía de respuestas entre poblaciones con diferencias en historia previa de exposición. El panorama de exposición previa a huracanes ha cambiado radicalmente en años recientes. Las estrategias de Macrodatos (Big Data) pudiesen ayudar a dilucidar estas dinámicas, lo cual podría ser instrumental en la adaptación de los planes de preparación para emergencias y para el diseño efectivo de estrategias de primeros auxilios de salud mental. 


\section{Introduction}

Tropical cyclones (TCs), most known as hurricanes, are one of the most devastating natural-occurring phenomena. They are the most damaging natural disaster in the US ${ }^{1}$. In recent times, it is apparent that TCs have been hitting landfall at much higher intensities than in previous years. To quantify how much more intense TCs have been, the power dissipation index (PDI), serves as a measurement of how destructive a TC is. Through this work, it is shown that the PDI for TCs has been increasing significantly since the 1970s (Emmanuel, 2005). Additionally, sea surface temperatures (SSTs), which have been increasing as a result of global warming ${ }^{2}$, strongly correlate positively to PDI values for TCs in the Atlantic region. Later, Emanuel (2007) went on to determine that additional factors contribute to the PDI, all of which are simulated by global climate models and can be used to project PDI values. Work done by Knutson et al. (2013), for example, further confirms that increasing SSTs leads to higher PDI values. In short, TCs have been, and will continue to be, much more intense because of global warming.

Worth noting, however, is that the frequency of TCs in the Atlantic region is expected to remain the same. Knutson et al. (2013) remarked among their conclusions that, based on their projections, an overall decrease in TC frequency should be expected in the Atlantic region. ${ }^{3}$ However, Vecchi et al, (2013) present in their work examples of studies with conflicting conclusions ${ }^{5}$; that is, some project an increase in frequency of TCs while others present a decrease. Work followed up by Murakami et al. (2014) for example, conclude that further development of predictive models is required to create more robust predictions regarding the frequency of TCs in the Atlantic region.

Before the 2017 Hurricane season, most nations in the Caribbean had been spared in recent years of significant hurricane damage. However, this rapidly changed beginning with Hurricane's Irma and María of the 2017 Season and has continued with 2019's Hurricane Dorian being the most recent instance (Colón-Zayas, 2018). Natural disasters are an unpredictable and uncontrollable source of anxiety we believe represent a prime candidate for symptomatic onset (Kar, et al. 2006; Orengo-Aguayo, et al. 2019). We posit that the manifested social media response will reflect the level of disaster preparedness of the population, which will vary in function of their recent exposure history and facilitate population level public health monitoring (De Choudhury, 2013).

Language is the social fabric that underlies thoughts, feelings, and emotions, which in turn guide our behavior (Rude, et al. 2004). The choice of words we use to express ourselves are not arbitrary or accidental, but the contrary. The ways people use words in their daily lives can provide rich information about their beliefs, fears, thinking patterns, social relationships, and personalities (Tausczik \& Pennebaker, 2010). In other words, through language use we can access our internal thoughts and emotions into a form that others can understand. This enables us to parse what is considered normal or abnormal in the psychopathology spectrum.

Our current paradigm of diagnosis of mental disorders is limited to language-based assessments in the form of clinical criteria. If the content of what we say is symptomatically loaded, correlations can be drawn as to what that person in particular is experiencing. Although it's been the clinician's job to detect what is symptomatically salient, linguistically speaking, using computer-based text analysis, a whole avenue of large corpuses can be addressed and evaluated through a computational linguistics lens (Mazuz and Yom-Tov, 2020).

Social media applications contain huge volumes of text generated by users themselves. In recent years, researchers have begun to explore linguistic signals deemed relevant of clinical content in internet forums, social media and other online communities. (see, Nguyen et al., 2014; Shen \& Rudzicz, 2017). For example, for depression specific symptomatology, not only the content of language (ie. negative valanced words) seemed to be predictive of depression, but also the way or style these people use function words (ie. pronouns, prepositions, etc.) (Rude et al., 2004). Another study found that the use of 'absolute words', and hence, 'absolutist thinking', was indicative of anxiety and depression symptomatology, as well as suicidal ideation in the context of online communities, which would lead to cognitive distortions as established in cognitive-based psychotherapies (Al-Mosaiwi \& Johnstone, 2018).

However, application of these approaches to reactions to on-going events has been limited. Though common in the analysis of political discourse, and polarization throughout bipartisan battles, more generalized examples of situational stress have been less commonly examined (Gaspar, et. al, 2016). Therefore, many questions remain on how social network analysis could be implemented beyond politics or binary sentiment. This report lays the foundation to initiate this analysis in the context of the Caribbean and the content dynamics elucidated during the 2019 Atlantic Hurricane Season. 


\section{Methods}

\section{Data collection}

Data was collected from two main sources: the National Oceanic and Atmospheric Administration (NOAA) National Hurricane Center (NHC) and Twitter. Digital historic records of hurricane trajectory and intensity were recollected from 2010 onwards by accessing the records of the NHC (NOAA, 2020). For the duration of the 2019 Hurricane Season, daily tweets where any of the names of the Atlantic Named-Storms was mentioned were sampled as permitted by the Twitter API through Rtweet and stored for future analysis (Kearney, 2019).

\section{Historical hurricane exposure}

Utilizing the digital records supplied by the National Hurricane service, each geographically and politically contiguous territory in the Caribbean was registered for their minimal proximity to each hurricane, and their intensity at this minimal proximity.

\section{Data processing-Baseline substraction}

Data collected for each major hurricane could vary largely. Dependent on the name, certain collections widely encompassed largely irrelevant material. For example, for Hurricane Karen, there was a large amount of background noise, as Karen is a common name used by many Twitter users. On the other hand, Hurricane Dorian suffered less from this contamination. To remediate this, baseline tweet count levels were calculated, through gaussian fit minimization.

\section{Results}

\section{History of Major Hurricanes in the Caribbean}

Table 1 presents summary statistics for 10 of the Major Hurricanes studied. Data as far back as 2010 in supplementary information. This includes dates and location for formation, peak intensity and dissipation, as well as code and storm name.

Figure $1 \mathrm{~A}$. Change in exposure to recent Hurricane activity in the Caribbean. illustrates territories with major hurricane exposure history up until the ending of the 2016 Hurricane Season.
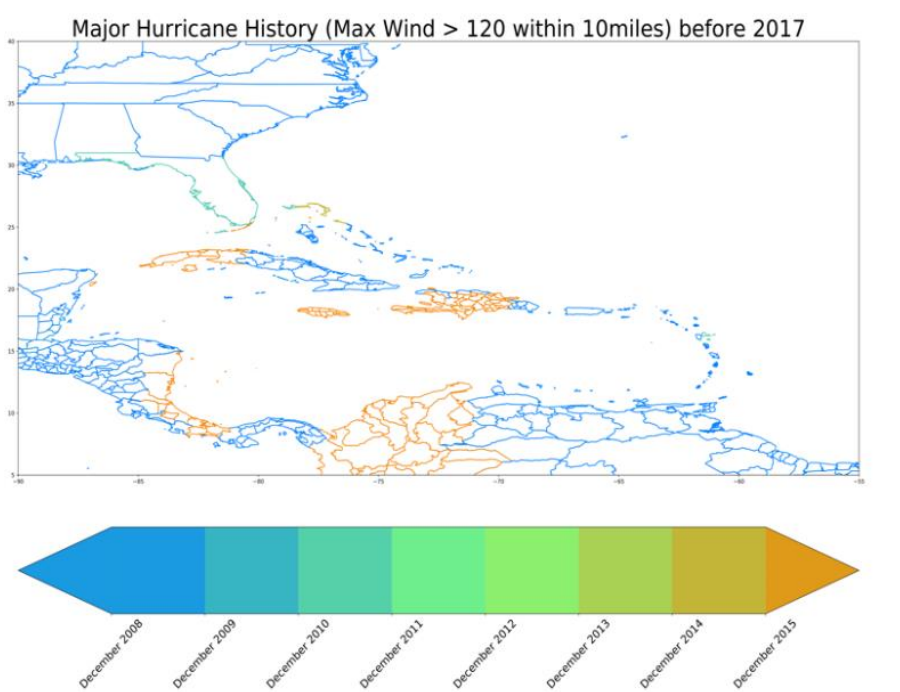

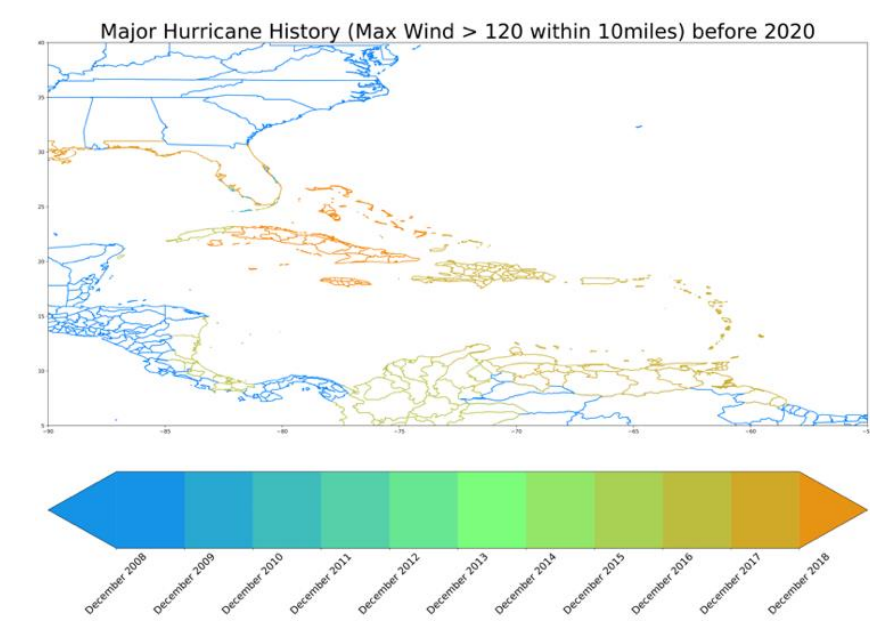

Figure 1 B. Change in exposure to recent Hurricane activity in the Caribbean. illustrates territories with major hurricane exposure history up until the ending of the 2019 Hurricane Season.

With this data, the history of hurricane exposure by territory is presented in Figure 1, demonstrating a rapid change in exposure history over the last few years

\section{Storm of Note}

As can be shown from the historical analysis captured in Figure 1, the distribution of historical hurricane exposure has been in rapid change in the Caribbean over the past few years.

Puerto Rico and the Abaco Islands, historically largely spared from major Hurricane activity prior to 2017, have now both recently been impacted by major storms. Figure 2, captures some significant differences between what we can denote as 3 particularly interesting storms, with 2 directly related to these 2 territories. The first storm of note is Hurricane Dorian, an extremely powerful storm that impacted the Bahamas, particularly the Abaco Islands, very closely. The second storm, Hurricane Karen, threatened Puerto Rico, however never experienced major intensificiation. The third storm, Hurricane Lorenzo, one of the most powerful storms we have observed so early in the Atlantic, formed and dissipated without much landfall anywhere at all.

Figure 2. Comparison framework for intensity and proximity. Dissection of the 2019

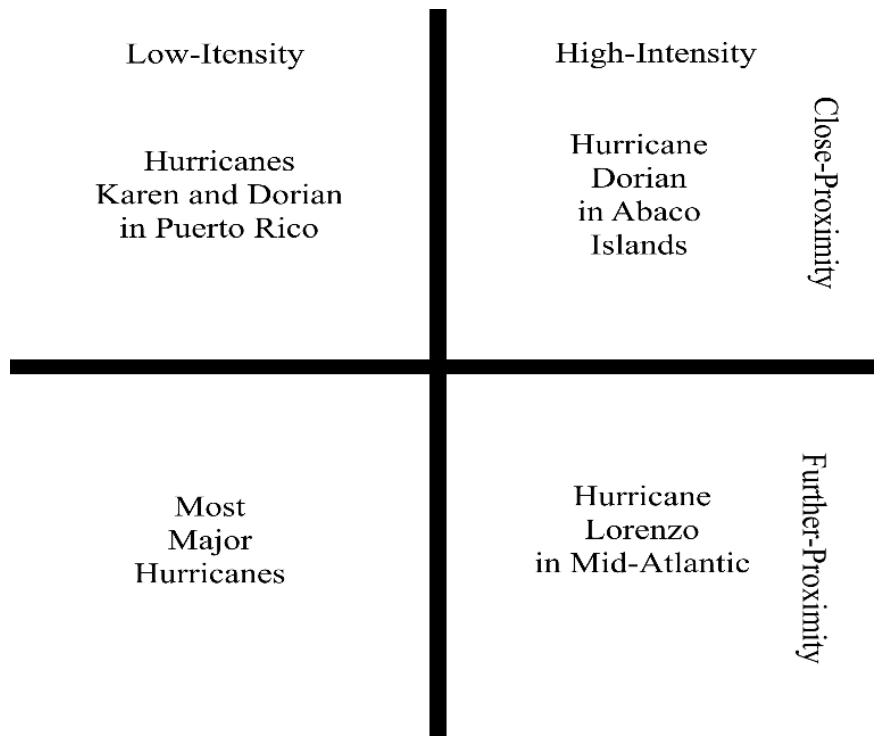




\section{Excess Determination}

Focusing on the three storms of note identified, baseline activity was determined beyond the confines of immediate storm activity. Figure 3 contains visualizations of the peak and baseline detection process, and Table 2 summarizes the outcome of this process by comparing baseline tweet activity, peak activity, and total activity before and after excess estimation.
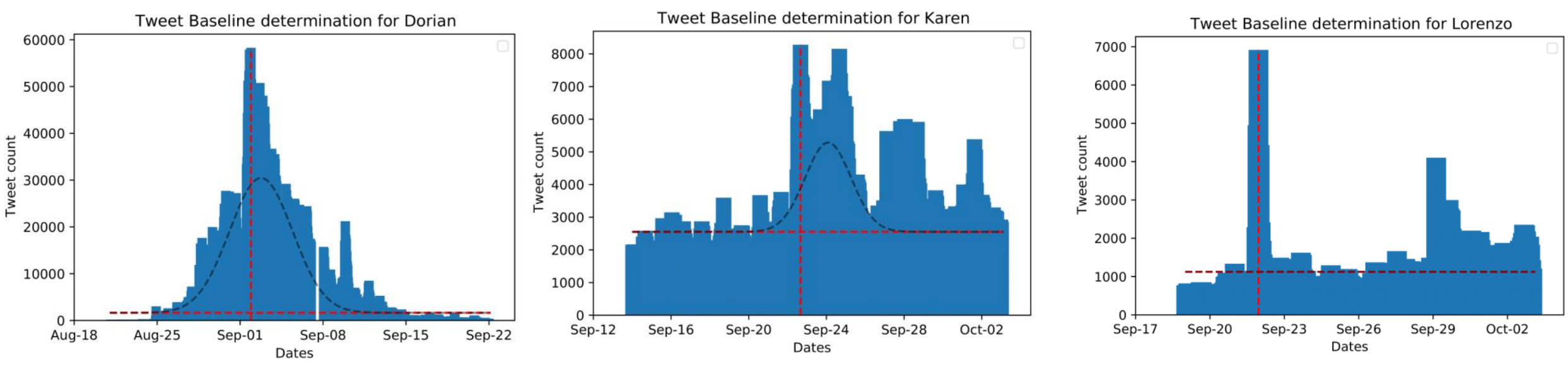

Figure 1. Baseline tweet determination for each storm. Each panel illustrates the highest hourly tweet count, and the baseline as determine by optimal Gaussian fitting for each Storm.

\section{Interaction of Tweet Activity, Hurricane Intensity and Proximity}

Statistical analysis included the construction of General Linear Models to explore the influence of two different independent variables: intensity and proximity, on one continuous dependent variable, tweet activity after excess filtering. Results tabulated in Table 3 and visualized in Figure 4.
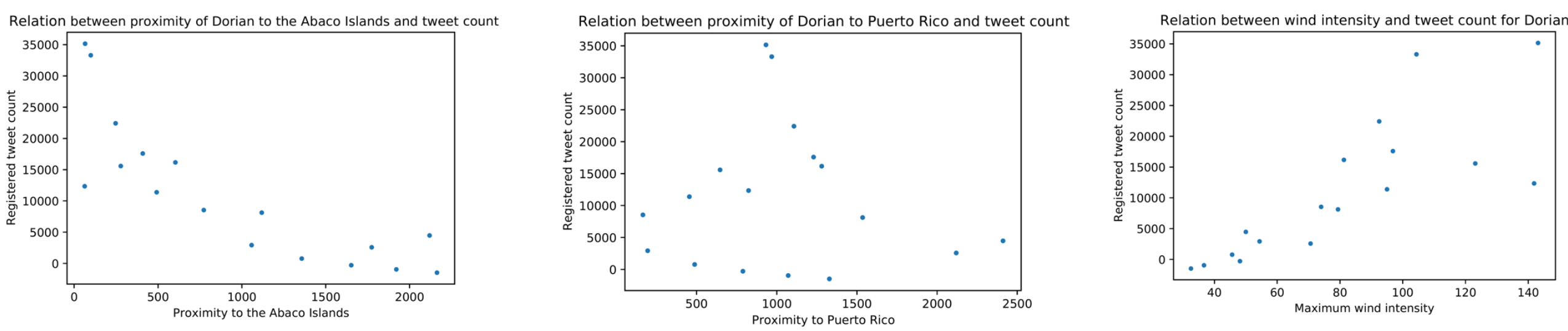


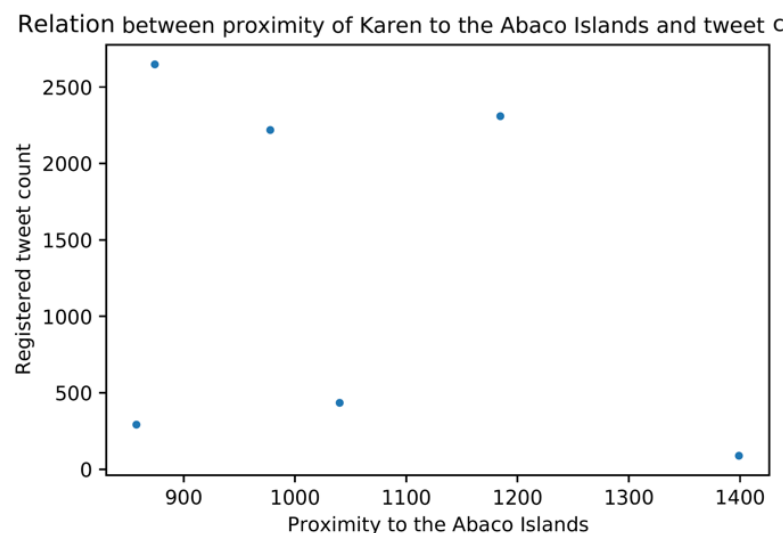

Relation between proximity of Lorenzo to the Abaco Islands and tweet count

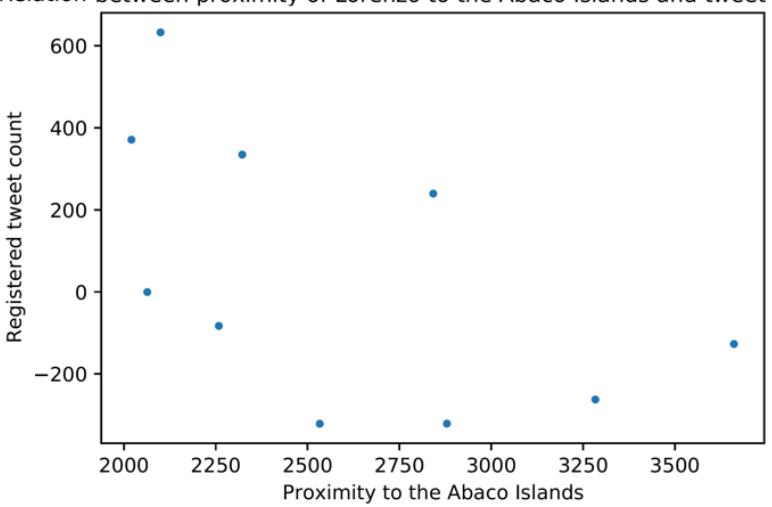

Relation between proximity of Karen to Puerto Rico and tweet count

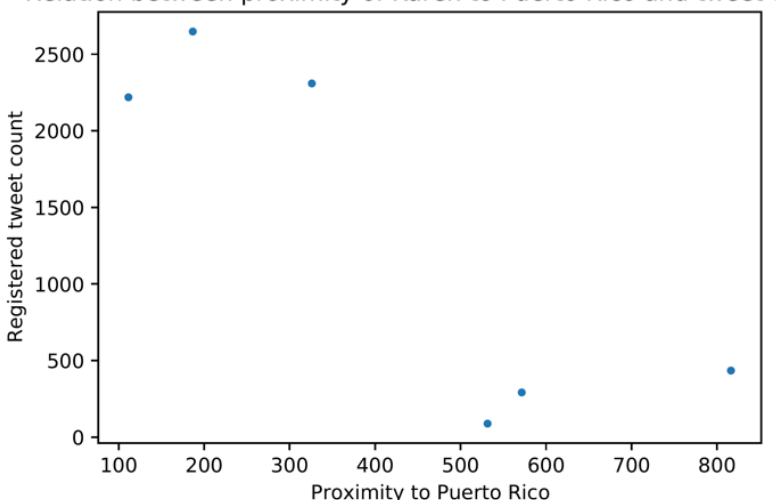

Relation between proximity of Lorenzo to Puerto Rico and tweet count

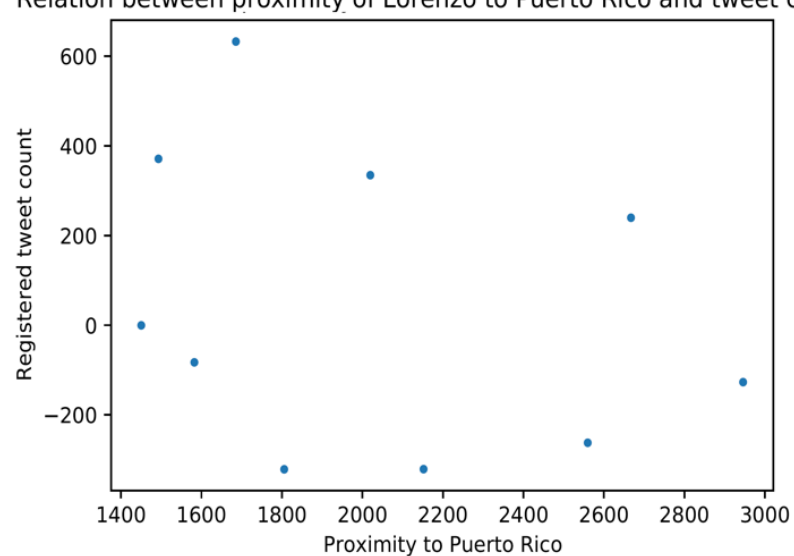

Relation between wind intensity and tweet count for Karen

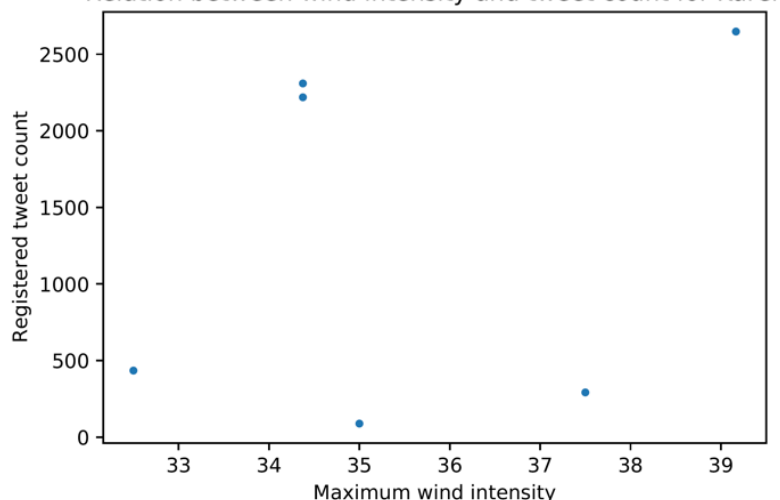

Relation between wind intensity and tweet count for Lorenzo

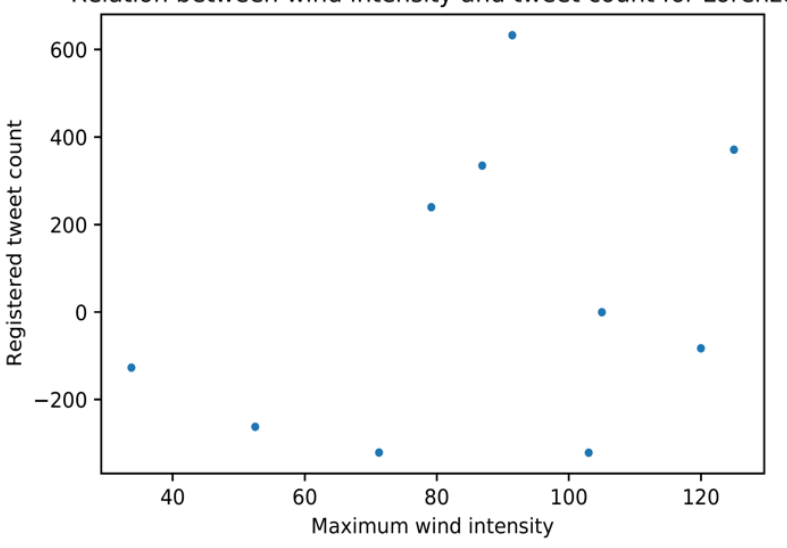

Figure 2 Multiple comparisons of proximity and intensity with Social Media response. One dimensional illustration of relationship between these variables for each location and storm of interest. 


\section{Discusion}

As captured in Figure 1, the landscape of recent major hurricane exposure in the Caribbean has rapidly changed in the last few years. The extensive repercussions this has brought upon their local health, economic and social infrastructure has been amply documented in the literature (Amadeo, 2020; Doran, et al. 2019). However, this visualization leaves patent how recent the extent of hurricane damage has been across the entire Caribbean.

As we inspect the 10 strongest hurricanes we have had in recent history, summarized in Table 1, we can immediately note that two of them were observed in the 2019 Hurricane Season. This led to their immediate inclusion in our following up inspection of Social Media data. Additionally, data for Hurricane Karen was also included, given how close this Hurricane was to impacting Puerto Rico, an island still struggling with recovery from its last major hurricane impact.

This arrangement allows us to parse these 3 major storms in two main domains: intensity and proximity. Most major storms, thankfully, are low intensity and remain at a distance from most human-inhabited territory. However, among these 3 storms, we can see how they deviate from the norm in distinct ways. Hurricane Karen, despite not registering major intensification, did make a close approach to Puerto Rico. On the other hand, Hurricane Lorenzo intensified significantly, but remained in the mid-Atlantic, posing no major threat. Finally, Hurricane Dorian both intensified significantly, and caused widespread damage, particularly in the Abaco Islands. This classification scheme is what is illustrated in Figure 2 and establishes the foundation for a framework in which to interpret the statistical differences observed during their trajectory.

After correction for baseline activity, we can see in the distinct windows of Figure 4 that low-dimensional 1 to 1 relationships between these variables do seem to carry some relation. This low-level inspection already heavily suggests that, nor intensity nor proximity are the definite arbiters of Social Media activity. On the contrary, the interaction effect between these reveals that it is their summative presence that potentiates the sensitization of the social media response. We can find $\mathrm{p}$-values for each coefficient in a GLM fixed for each Hurricane and location in Table 3. Hurricane Lorenzo, having never approached the Caribbean made comparison in proximity a moot point, for which reason results are only presented inspecting the relation of its intensity with Social Media Activity. Nonetheless, moving beyond just a discussion of alpha levels, inspecting these $p$-values is informative of the dynamic influence the interaction effect truly has. For example, we can, at an alpha level of 0.10 , arbitrarily remark that intensity was statistically significant for Tweet Counts generated by Dorian in Puerto Rico, but not the Bahamas. However, it is of greater interest to observe that, beyond alpha levels, the influence of proximity was equally dynamic, changing between the datapoint of interest, while the interaction
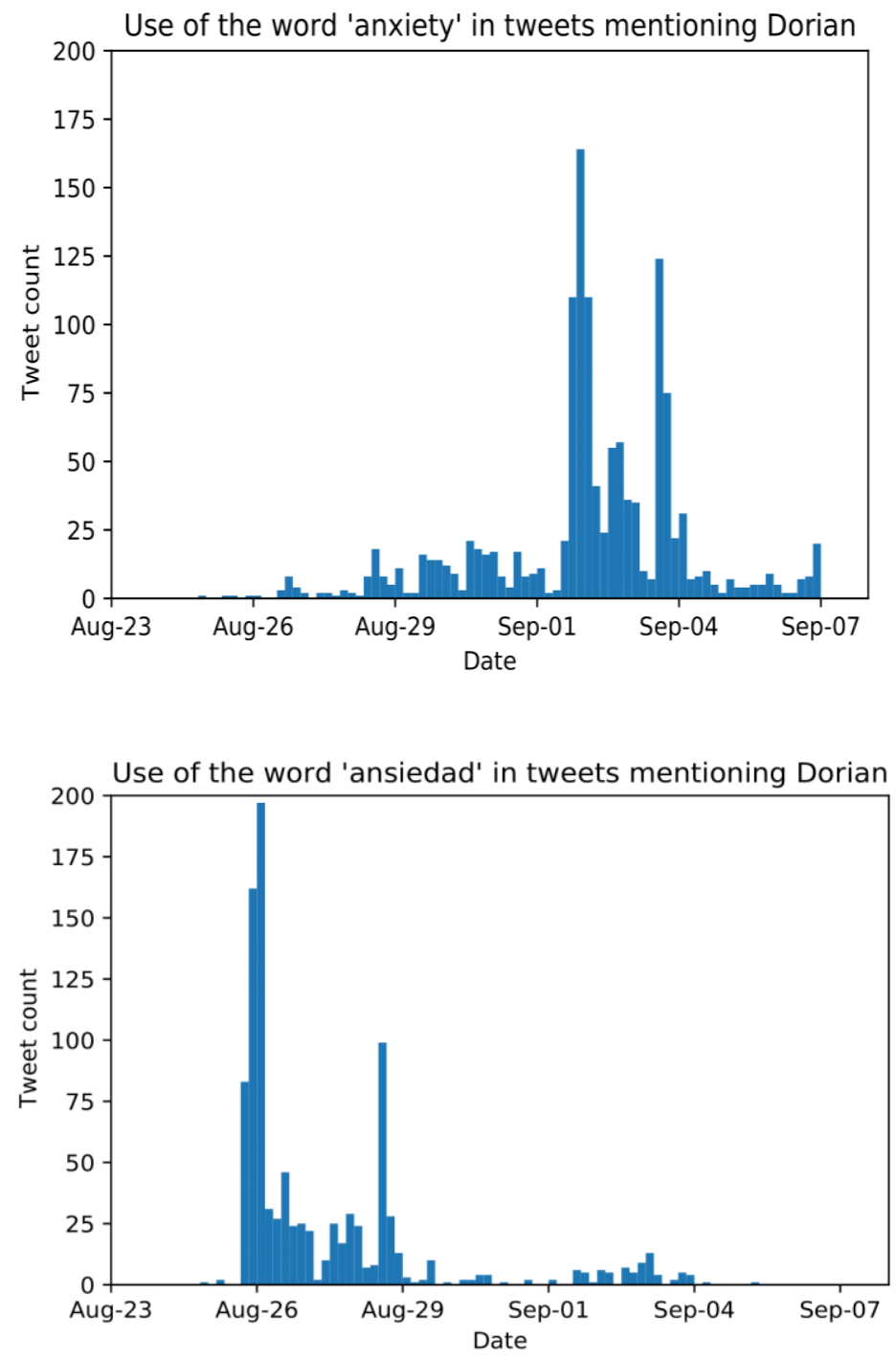

Figure 3 Contrast in usage of Anxiety and 'Ansiedad' during Hurricane Dorian. Visualization of the clear time-domain separation of word usage on Social Media, reflective of underlying geographical and linguistic patterns. 
effect, commonly allowed higher alpha levels in the literature, remains stable.

Therefore, Hurricane Dorian presented the opportunity of evaluating the continued Social Media Response in two contexts: on approach to Puerto Rico and on approach to the Abaco Islands. To further explore the differences in these two contexts, future studies could explore content level differences. We exemplify this with a simple contrast between the usage of words "Ansiedad" and "Anxiety", synonyms in Spanish and English respectively that denote a feeling of unease, such as worry or fear, that can be mild or severe and carry clinical connotations, throughout the course of Dorian in Figure 5. We note that these distributions are markedly different, with "Ansiedad" strongly reflecting the fact that, Hurricane Dorian menaced Spanishspeaking Caribbean nations first, before finally devastating the English-speaking populations of the Bahamas, and these two separate populations seem to be the main drivers of the activity at each peak. We would suggest the implementation of nonnegative matrix factorization to identify the content-level relevant material, to potentiate the dimensions of linguistic criteria that could be compared in the future (Dao, et al. 2016).

\section{Acknowledgements}

We would like to thank Andrés Josué Arroyo and Ileana Ayala Fontánez for their commentary and input on work, and our donors who contributed to our work via Ko-Fi.com

\section{Literature cited}

[1] Amadeo, K. 2020. How Hurricanes Damage the Economy. The Balance [Online]. https://www.thebalance.com/cost-of-naturaldisasters-3306214

[2] Al-Mosaiwi, M., and Johnstone, T. 2018. In an absolute state: Elevated use of absolutist words is a marker specific to anxiety, depression, and suicidal ideation. Clinical Psychological Science, 6(4), 529-542.

[3] Colón-Zayas, E. 2018. Puerto Rico en tiempos del huracán María o Puerto Rico entre laboratorio económico y estrategias militares, los azotes y el amparo del huracán María. Revista de Estudios Internacionales, 3(5), 115-124.

[4] Dao, B., Nguyen, T., Venkatesh, S. and Phung, D. 2016. Discovering latent affective dynamics among individuals in online mental health-related communities. Proceedings of the Internal Conference on Multimedia.

[5] De Choudhury, M. 2013. Role of social media in tackling challenges in mental health. Proceedings of the 2nd International

[6] Conference on Education and Multimedia Technology, 49-52. doi:10.1145/2509916.2509921.

[7] Doran, C. M. \& Kinchin, I. 2019. A review of the economic impact of mental illness. Australian Health Review. 43 , 43-48.

[8] Emanuel, K. 2005. Increasing destructiveness of tropical cyclones over the past 30 years. Nature, 436(7051), 686688. doi:10.1038/nature 03906

[9] Emanuel, K. 2007. Environmental Factors Affecting Tropical Cyclone Power Dissipation. Journal of Climate, 20(22), 54975509. doi:10.1175/2007jcli1571.1

[10] Gaspar, R., Pedro, C., Panagiotopoulos, P. and Seibt, B. 2016. Beyond positive or negative: Qualitative sentiment analysis of social media reactions to unexpected stressful events. Computational Human Behavior. 56, 179-191

[11] Kar, N., Kumar Bastia, B., Kumar, B., and Associate, B. 2006. Post-traumatic stress disorder, depression and generalised anxiety disorder in adolescents after a natural disaster: a study of comorbidity. doi:10.1186/1745-0179-2.

[12] Knutson, T. R., Sirutis, J. J., Vecchi, G. A., Garner, S., Zhao, M., Kim, H.-S., and Villarini, G. 2013. Dynamical Downscaling Projections of Twenty-First-Century Atlantic Hurricane Activity: CMIP3 and CMIP5 Model-Based Scenarios. Journal of Climate, 26(17), 6591-6617. doi:10.1175/jcli-d-12-00539.1

[13] Mazuz, K., and Yom-Tov, E. 2020. Analyzing Trends of Loneliness Through Large-Scale Analysis of Social Media Postings: Observational Study. Journal of Medical Internet Research. 7(4):e17188 https://www.ncbi.nlm.nih.gov/pubmed/32310141

[14] Murakami, H., Hsu, P.-C., Arakawa, O., and Li, T. 2014. Influence of Model Biases on Projected Future Changes in Tropical Cyclone Frequency of Occurrence. Journal of Climate, 27(5), 2159-2181. doi:10.1175/jcli-d-13-00436.1

[15] National Oceanic and Atmospheric Administration. 2020. National Hurricane Center Data Archive. Accessed at https://www.nhc.noaa.gov/data/.

[16] Nguyen, T., Phung, D., Dao, B., Venkatesh, S., and Berk, M. 2014. Affective and content analysis of online depression communities. Transactions on Affective Computing, 5(3), 217-226.

[17] Orengo-Aguayo, R., Stewart, R. W., de Arellano, M. A., Suárez-Kindy, J. L., and Young, J. 2019. Disaster Exposure and Mental Health Among Puerto Rican Youths After Hurricane Maria. JAMA network open, 2(4), e192619.

https://doi.org/10.1001/jamanetworkopen.2019.2619

[18] Rude, S., Gortner, E. M., and Pennebaker, J. 2004. Language use of depressed and depression-vulnerable college students. Cognition \& Emotion, 18(8), 1121-1133. 
[19] Shen, J. H., and Rudzicz, F. 2017. Detecting anxiety through reddit. Proceedings of the Fourth Workshop on Computational Linguistics and Clinical Psychology-From Linguistic Signal to Clinical Reality (pp. 58-65).

[20] Tausczik, Y. R., and Pennebaker, J. W. (2010). The psychological meaning of words: LIWC and computerized text analysis methods. Journal of language and social psychology, 29(1), 24-54.

[21] Vecchi, G. A., Msadek, R., Anderson, W., Chang, Y.-S., Delworth, T., Dixon, K., ... Zhang, S. (2013). Multiyear Predictions of North Atlantic Hurricane Frequency: Promise and Limitations. Journal of Climate, 26(15), 5337-5357. doi:10.1175/jcli-d-12-00464.1 
Table 1. Major Atlantic Storm History. Data extracted from the National Hurricane Center historical archive.

\begin{tabular}{|c|c|c|c|c|c|c|c|c|}
\hline \multirow{2}{*}{$\begin{array}{c}\text { Code } \\
\text { al052019 }\end{array}$} & \multirow{2}{*}{$\begin{array}{c}\text { Name } \\
\text { Hurricane Dorian }\end{array}$} & \multicolumn{2}{|c|}{$\begin{array}{l}\text { Formation Date and } \\
\text { Coordinates }\end{array}$} & \multicolumn{2}{|c|}{$\begin{array}{l}\text { Dissipation Date and } \\
\text { Coordinates }\end{array}$} & \multicolumn{3}{|c|}{$\begin{array}{c}\text { Max Wind Intensity, Date and } \\
\text { Coordinates }\end{array}$} \\
\hline & & $\begin{array}{c}8 / 24 / 2019 \\
15: 00\end{array}$ & $\begin{array}{c}{[-47.9} \\
10.4]\end{array}$ & 9/9/2019 3:00 & $\begin{array}{c}{[-53.4} \\
52.1]\end{array}$ & 160 & 9/1/2019 21:00 & $\begin{array}{c}{[-77.3,} \\
26.6]\end{array}$ \\
\hline al112017 & Hurricane Irma & $\begin{array}{c}8 / 30 / 2017 \\
15: 00\end{array}$ & $\begin{array}{c}{[-30.3} \\
16.4]\end{array}$ & $\begin{array}{c}9 / 12 / 2017 \\
3: 00\end{array}$ & $\begin{array}{c}{[-84.9} \\
32.4]\end{array}$ & 160 & 9/6/2017 12:00 & $\begin{array}{c}{[-63.3,} \\
18.1]\end{array}$ \\
\hline al152017 & Hurricane Maria & $\begin{array}{c}9 / 16 / 2017 \\
15: 00\end{array}$ & $\begin{array}{c}{[-50.5,} \\
12.2]\end{array}$ & $\begin{array}{c}9 / 30 / 2017 \\
21: 00\end{array}$ & $\begin{array}{c}{[-43.9,} \\
42.0]\end{array}$ & 150 & 9/20/2017 3:00 & $\begin{array}{c}{[-64.7,} \\
17.3]\end{array}$ \\
\hline al132019 & Hurricane Lorenzo & $\begin{array}{c}9 / 23 / 2019 \\
3: 00\end{array}$ & $\begin{array}{c}{[-20.9} \\
10.8]\end{array}$ & $\begin{array}{c}10 / 2 / 2019 \\
15: 00\end{array}$ & $\begin{array}{c}{[-25.8,} \\
44.4]\end{array}$ & 140 & 9/29/2019 3:00 & $\begin{array}{c}{[-44.9,} \\
24.2]\end{array}$ \\
\hline al142016 & Hurricane Matthew & $\begin{array}{c}9 / 28 / 2016 \\
15: 00\end{array}$ & $\begin{array}{c}{[-60.7} \\
13.4]\end{array}$ & $\begin{array}{c}10 / 9 / 2016 \\
21: 00\end{array}$ & $\begin{array}{c}{[-72.0} \\
35.4]\end{array}$ & 140 & 10/1/2016 3:00 & $\begin{array}{c}{[-72.3,} \\
13.3]\end{array}$ \\
\hline al112010 & Hurricane Igor & $\begin{array}{c}9 / 8 / 2010 \\
15: 00\end{array}$ & $\begin{array}{c}{[-23.5,} \\
13.7]\end{array}$ & $\begin{array}{c}9 / 21 / 2010 \\
21: 00\end{array}$ & $\begin{array}{c}{[-51.7} \\
49.3]\end{array}$ & 135 & 9/15/2010 3:00 & $\begin{array}{c}{[-53.9,} \\
19.0]\end{array}$ \\
\hline al112015 & Hurricane Joaquin & $\begin{array}{c}9 / 28 / 2015 \\
3: 00\end{array}$ & $\begin{array}{c}{[-68.7,} \\
27.5]\end{array}$ & $\begin{array}{c}10 / 8 / 2015 \\
3: 00\end{array}$ & $\begin{array}{c}{[-37.0} \\
42.0]\end{array}$ & 135 & $\begin{array}{c}10 / 3 / 2015 \\
18: 00\end{array}$ & $\begin{array}{c}{[-70.9,} \\
26.4]\end{array}$ \\
\hline al122017 & Hurricane Jose & $\begin{array}{c}9 / 5 / 2017 \\
15: 00\end{array}$ & $\begin{array}{c}{[-39.1} \\
12.3]\end{array}$ & $\begin{array}{c}9 / 22 / 2017 \\
21: 00\end{array}$ & $\begin{array}{c}{[-69.1} \\
39.3]\end{array}$ & 135 & 9/9/2017 3:00 & $\begin{array}{c}{[-59.3,} \\
16.9]\end{array}$ \\
\hline al142018 & Hurricane Michael & $\begin{array}{c}10 / 6 / 2018 \\
21: 00\end{array}$ & $\begin{array}{c}{[-86.6,} \\
18.0]\end{array}$ & $\begin{array}{c}10 / 12 / 2018 \\
9: 00\end{array}$ & $\begin{array}{c}{[-73.1} \\
38.0]\end{array}$ & 135 & $\begin{array}{c}10 / 10 / 2018 \\
18: 00\end{array}$ & $\begin{array}{c}{[-85.5,} \\
30.0]\end{array}$ \\
\hline al072008 & Hurricane Gustav & $\begin{array}{c}8 / 25 / 2008 \\
15: 00\end{array}$ & $\begin{array}{c}{[-70.1} \\
15.5]\end{array}$ & 9/2/2008 9:00 & $\begin{array}{c}{[-93.4,} \\
31.7]\end{array}$ & 130 & $8 / 31 / 20080: 00$ & $\begin{array}{c}{[-83.4,} \\
22.7]\end{array}$ \\
\hline al072010 & Hurricane Earl & $\begin{array}{c}8 / 25 / 2010 \\
15: 00\end{array}$ & $\begin{array}{c}{[-30.8} \\
14.3]\end{array}$ & 9/5/2010 3:00 & $\begin{array}{c}{[-59.2} \\
50.7]\end{array}$ & 126 & $9 / 2 / 201012: 00$ & $\begin{array}{c}{[-74.8,} \\
30.1]\end{array}$ \\
\hline al082014 & Hurricane Gonzalo & $\begin{array}{c}10 / 12 / 2014 \\
17: 30\end{array}$ & $\begin{array}{c}{[-58.4,} \\
16.4]\end{array}$ & $\begin{array}{c}10 / 19 / 2014 \\
21: 00\end{array}$ & $\begin{array}{c}{[-41.8,} \\
51.6]\end{array}$ & 126 & $\begin{array}{c}10 / 17 / 2014 \\
0: 00\end{array}$ & $\begin{array}{c}{[-67.8,} \\
27.4]\end{array}$ \\
\hline al092008 & Hurricane Ike & $\begin{array}{c}9 / 1 / 2008 \\
15: 00\end{array}$ & $\begin{array}{c}{[-39.5} \\
17.6]\end{array}$ & $\begin{array}{c}9 / 14 / 2008 \\
9: 00\end{array}$ & $\begin{array}{c}{[-92.5} \\
36.4]\end{array}$ & 125 & 9/4/2008 9:00 & $\begin{array}{c}{[-55.8,} \\
22.7]\end{array}$ \\
\hline al172008 & Hurricane Paloma & $\begin{array}{c}11 / 5 / 2008 \\
21: 00\end{array}$ & $\begin{array}{c}{[-81.8,} \\
14.0]\end{array}$ & $\begin{array}{c}11 / 10 / 2008 \\
3: 00\end{array}$ & $\begin{array}{c}{[-78.0,} \\
22.0]\end{array}$ & 125 & $\begin{array}{c}11 / 8 / 2008 \\
21: 00\end{array}$ & $\begin{array}{c}{[-78.5,} \\
20.5]\end{array}$ \\
\hline al162011 & Hurricane Ophelia & $\begin{array}{l}9 / 21 / 2011 \\
3: 00\end{array}$ & $\begin{array}{c}{[-40.1} \\
12.2]\end{array}$ & $\begin{array}{c}10 / 3 / 2011 \\
15: 00\end{array}$ & $\begin{array}{c}{[-52.0} \\
48.1]\end{array}$ & 122 & 10/2/2011 6:00 & $\begin{array}{r}{[-62.0,} \\
34.9]\end{array}$ \\
\hline
\end{tabular}




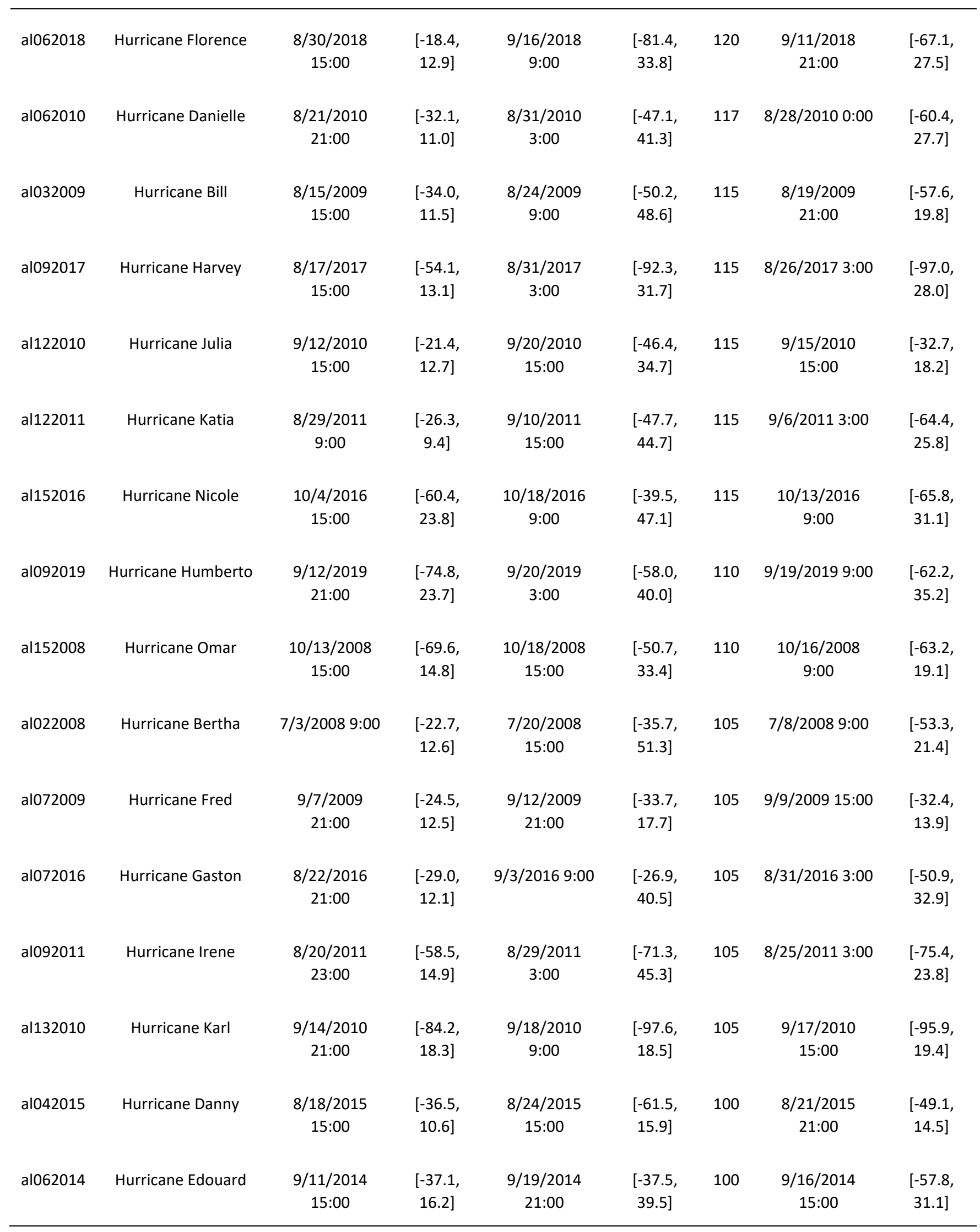




\begin{tabular}{|c|c|c|c|c|c|c|c|c|}
\hline al132012 & Hurricane Michael & $\begin{array}{c}9 / 3 / 2012 \\
21: 00\end{array}$ & $\begin{array}{c}{[-42.2} \\
25.6]\end{array}$ & $\begin{array}{c}9 / 11 / 2012 \\
21: 00\end{array}$ & $\begin{array}{c}{[-45.8} \\
41.4]\end{array}$ & 100 & 9/6/2012 15:00 & $\begin{array}{c}{[-41.3,} \\
30.1]\end{array}$ \\
\hline al142017 & Hurricane Lee & $\begin{array}{c}9 / 15 / 2017 \\
3: 00\end{array}$ & $\begin{array}{c}{[-25.4,} \\
10.7]\end{array}$ & $\begin{array}{c}9 / 30 / 2017 \\
9: 00\end{array}$ & $\begin{array}{c}{[-35.6} \\
46.7]\end{array}$ & 100 & $\begin{array}{c}9 / 27 / 2017 \\
21: 00\end{array}$ & $\begin{array}{c}{[-57.1,} \\
31.2]\end{array}$ \\
\hline al172017 & Hurricane Ophelia & $\begin{array}{c}10 / 9 / 2017 \\
9: 00\end{array}$ & $\begin{array}{c}{[-39.9} \\
31.1]\end{array}$ & $\begin{array}{c}10 / 16 / 2017 \\
3: 00\end{array}$ & $\begin{array}{c}{[-13.3} \\
49.2]\end{array}$ & 100 & $\begin{array}{c}10 / 14 / 2017 \\
21: 00\end{array}$ & $\begin{array}{c}{[-23.7,} \\
35.9]\end{array}$ \\
\hline al082012 & Hurricane Gordon & $\begin{array}{c}8 / 15 / 2012 \\
21: 00\end{array}$ & $\begin{array}{c}{[-55.1} \\
29.9]\end{array}$ & $\begin{array}{c}8 / 20 / 2012 \\
21: 00\end{array}$ & $\begin{array}{c}{[-20.3} \\
39.2]\end{array}$ & 96 & 8/19/2012 6:00 & $\begin{array}{c}{[-32.7,} \\
34.6]\end{array}$ \\
\hline al162016 & Hurricane Otto & $\begin{array}{c}11 / 21 / 2016 \\
9: 00\end{array}$ & $\begin{array}{c}{[-79.4,} \\
11.5]\end{array}$ & $\begin{array}{c}11 / 25 / 2016 \\
6: 00\end{array}$ & $\begin{array}{c}{[-86.2,} \\
10.8]\end{array}$ & 96 & $\begin{array}{c}11 / 24 / 2016 \\
18: 00\end{array}$ & $\begin{array}{c}{[-83.9,} \\
11.0]\end{array}$ \\
\hline al182011 & Hurricane Rina & $\begin{array}{c}10 / 23 / 2011 \\
21: 00\end{array}$ & $\begin{array}{c}{[-81.9,} \\
15.9]\end{array}$ & $\begin{array}{c}10 / 28 / 2011 \\
21: 00\end{array}$ & $\begin{array}{c}{[-86.1} \\
21.9]\end{array}$ & 96 & $\begin{array}{c}10 / 26 / 2011 \\
6: 00\end{array}$ & $\begin{array}{c}{[-85.0,} \\
17.5]\end{array}$ \\
\hline al182012 & Hurricane Sandy & $\begin{array}{c}10 / 22 / 2012 \\
15: 00\end{array}$ & $\begin{array}{c}{[-78.0} \\
13.5]\end{array}$ & $\begin{array}{c}10 / 30 / 2012 \\
3: 00\end{array}$ & $\begin{array}{c}{[-75.4} \\
39.8]\end{array}$ & 96 & $\begin{array}{c}10 / 25 / 2012 \\
6: 00\end{array}$ & $\begin{array}{c}{[-75.9} \\
20.1]\end{array}$ \\
\hline al082018 & Hurricane Helene & $\begin{array}{c}9 / 7 / 2018 \\
15: 00\end{array}$ & $\begin{array}{c}{[-17.8,} \\
13.1]\end{array}$ & $\begin{array}{c}9 / 16 / 2018 \\
15: 00\end{array}$ & $\begin{array}{c}{[-25.7,} \\
43.8]\end{array}$ & 95 & 9/11/2018 9:00 & $\begin{array}{c}{[-33.6,} \\
16.0]\end{array}$ \\
\hline al112009 & Hurricane Ida & $\begin{array}{c}11 / 4 / 2009 \\
15: 00\end{array}$ & $\begin{array}{c}{[-82.0} \\
11.6]\end{array}$ & $\begin{array}{c}11 / 10 / 2009 \\
15: 00\end{array}$ & $\begin{array}{c}{[-87.6} \\
30.6]\end{array}$ & 91 & 11/9/2009 6:00 & $\begin{array}{c}{[-87.5,} \\
24.4]\end{array}$ \\
\hline al032018 & Hurricane Chris & $\begin{array}{c}7 / 6 / 2018 \\
21: 00\end{array}$ & $\begin{array}{c}{[-73.8} \\
32.2]\end{array}$ & $\begin{array}{c}7 / 12 / 2018 \\
15: 00\end{array}$ & $\begin{array}{c}{[-57.7} \\
44.4]\end{array}$ & 90 & 7/11/2018 9:00 & $\begin{array}{c}{[-69.9,} \\
35.3]\end{array}$ \\
\hline al082017 & Hurricane Gert & $\begin{array}{c}8 / 13 / 2017 \\
3: 00\end{array}$ & $\begin{array}{c}{[-70.3,} \\
25.3]\end{array}$ & $\begin{array}{c}8 / 17 / 2017 \\
21: 00\end{array}$ & $\begin{array}{c}{[-46.0} \\
44.8]\end{array}$ & 90 & 8/17/2017 3:00 & $\begin{array}{c}{[-58.4,} \\
40.1]\end{array}$ \\
\hline al102019 & Hurricane Jerry & $\begin{array}{c}9 / 17 / 2019 \\
15: 00\end{array}$ & $\begin{array}{c}{[-44.9} \\
12.9]\end{array}$ & $\begin{array}{c}9 / 25 / 2019 \\
21: 00\end{array}$ & $\begin{array}{c}{[-65.6,} \\
32.2]\end{array}$ & 90 & 9/20/2019 6:00 & $\begin{array}{c}{[-57.9,} \\
18.1]\end{array}$ \\
\hline al112012 & Hurricane Kirk & $\begin{array}{c}8 / 28 / 2012 \\
21: 00\end{array}$ & $\begin{array}{c}{[-43.9} \\
23.8]\end{array}$ & $\begin{array}{c}9 / 2 / 2012 \\
21: 00\end{array}$ & $\begin{array}{c}{[-32.6} \\
49.7]\end{array}$ & 90 & $\begin{array}{c}8 / 31 / 2012 \\
15: 00\end{array}$ & $\begin{array}{c}{[-50.8,} \\
31.2]\end{array}$ \\
\hline al132017 & Hurricane Katia & $\begin{array}{c}9 / 5 / 2017 \\
21: 00\end{array}$ & $\begin{array}{c}{[-96.7} \\
22.4]\end{array}$ & $\begin{array}{c}9 / 9 / 2017 \\
12: 00\end{array}$ & $\begin{array}{c}{[-97.7} \\
20.1]\end{array}$ & 90 & 9/8/2017 21:00 & $\begin{array}{c}{[-96.5,} \\
21.0]\end{array}$ \\
\hline al162018 & Hurricane Oscar & $\begin{array}{c}10 / 27 / 2018 \\
3: 00\end{array}$ & $\begin{array}{c}{[-45.7} \\
26.7]\end{array}$ & $\begin{array}{c}10 / 31 / 2018 \\
21: 00\end{array}$ & $\begin{array}{c}{[-49.6} \\
39.3]\end{array}$ & 90 & $\begin{array}{c}10 / 30 / 2018 \\
9: 00\end{array}$ & $\begin{array}{c}{[-58.2,} \\
28.6]\end{array}$ \\
\hline al012010 & Hurricane Alex & $\begin{array}{c}6 / 25 / 2010 \\
22: 00\end{array}$ & $\begin{array}{c}{[-83.5} \\
16.5]\end{array}$ & 7/2/2010 3:00 & $\begin{array}{c}{[-102.4} \\
23.3]\end{array}$ & 87 & 7/1/2010 1:00 & $\begin{array}{c}{[-97.5,} \\
24.3]\end{array}$ \\
\hline
\end{tabular}




\begin{tabular}{|c|c|c|c|c|c|c|c|c|}
\hline al012014 & Hurricane Arthur & 7/1/2014 3:00 & $\begin{array}{c}{[-79.1} \\
27.6]\end{array}$ & $\begin{array}{c}7 / 5 / 2014 \\
15: 00\end{array}$ & $\begin{array}{c}{[-65.5,} \\
45.0]\end{array}$ & 87 & 7/4/2014 7:00 & $\begin{array}{c}{[-75.9,} \\
35.6]\end{array}$ \\
\hline al182010 & Hurricane Paula & $\begin{array}{c}10 / 11 / 2010 \\
21: 00\end{array}$ & $\begin{array}{c}{[-84.0,} \\
16.0]\end{array}$ & $\begin{array}{c}10 / 15 / 2010 \\
15: 00\end{array}$ & $\begin{array}{c}{[-79.5} \\
22.8]\end{array}$ & 87 & $\begin{array}{c}10 / 13 / 2010 \\
12: 00\end{array}$ & $\begin{array}{c}{[-85.9,} \\
21.3]\end{array}$ \\
\hline al212010 & Hurricane Tomas & $\begin{array}{c}10 / 29 / 2010 \\
21: 00\end{array}$ & $\begin{array}{c}{[-57.5} \\
11.1]\end{array}$ & $\begin{array}{c}11 / 7 / 2010 \\
21: 00\end{array}$ & $\begin{array}{c}{[-69.1} \\
26.1]\end{array}$ & 87 & $\begin{array}{c}10 / 31 / 2010 \\
12: 00\end{array}$ & $\begin{array}{c}{[-63.3,} \\
14.0]\end{array}$ \\
\hline al042008 & Hurricane Dolly & $\begin{array}{c}7 / 20 / 2008 \\
15: 45\end{array}$ & $\begin{array}{c}{[-84.2} \\
18.4]\end{array}$ & $\begin{array}{c}7 / 24 / 2008 \\
21: 00\end{array}$ & $\begin{array}{c}{[-100.6,} \\
28.2]\end{array}$ & 85 & $\begin{array}{c}7 / 23 / 2008 \\
17: 00\end{array}$ & $\begin{array}{c}{[-97.0,} \\
26.2]\end{array}$ \\
\hline al132018 & Hurricane Leslie & $\begin{array}{c}9 / 23 / 2018 \\
15: 00\end{array}$ & $\begin{array}{c}{[-46.5,} \\
33.0]\end{array}$ & $\begin{array}{c}10 / 13 / 2018 \\
21: 00\end{array}$ & $\begin{array}{l}{[-9.5} \\
40.5]\end{array}$ & 80 & $\begin{array}{c}10 / 12 / 2018 \\
9: 00\end{array}$ & $\begin{array}{c}{[-31.0,} \\
31.9]\end{array}$ \\
\hline al142012 & Hurricane Nadine & $\begin{array}{c}9 / 11 / 2012 \\
15: 00\end{array}$ & $\begin{array}{c}{[-43.1} \\
16.3]\end{array}$ & $\begin{array}{c}10 / 4 / 2012 \\
15: 00\end{array}$ & $\begin{array}{c}{[-26.7} \\
40.0]\end{array}$ & 80 & $\begin{array}{c}9 / 30 / 2012 \\
15: 00\end{array}$ & $\begin{array}{c}{[-38.6,} \\
37.1]\end{array}$ \\
\hline al162017 & Hurricane Nate & $\begin{array}{c}10 / 4 / 2017 \\
15: 00\end{array}$ & $\begin{array}{c}{[-81.9} \\
12.2]\end{array}$ & $\begin{array}{c}10 / 8 / 2017 \\
15: 00\end{array}$ & $\begin{array}{c}{[-87.3} \\
33.1]\end{array}$ & 80 & $\begin{array}{c}10 / 7 / 2017 \\
18: 00\end{array}$ & $\begin{array}{c}{[-88.9,} \\
27.6]\end{array}$ \\
\hline al172011 & Hurricane Philippe & $\begin{array}{c}9 / 24 / 2011 \\
9: 00\end{array}$ & $\begin{array}{c}{[-25.2,} \\
10.7]\end{array}$ & $\begin{array}{c}10 / 9 / 2011 \\
3: 00\end{array}$ & $\begin{array}{c}{[-43.9,} \\
38.4]\end{array}$ & 80 & 10/7/2011 9:00 & $\begin{array}{c}{[-56.3,} \\
29.6]\end{array}$ \\
\hline al172012 & Hurricane Rafael & $\begin{array}{c}10 / 12 / 2012 \\
23: 30\end{array}$ & $\begin{array}{c}{[-63.1} \\
15.0]\end{array}$ & $\begin{array}{c}10 / 17 / 2012 \\
21: 00\end{array}$ & $\begin{array}{c}{[-56.5} \\
40.2]\end{array}$ & 80 & $\begin{array}{c}10 / 16 / 2012 \\
15: 00\end{array}$ & $\begin{array}{c}{[-64.5,} \\
28.6]\end{array}$ \\
\hline al192010 & Hurricane Richard & $\begin{array}{c}10 / 21 / 2010 \\
3: 00\end{array}$ & $\begin{array}{c}{[-81.1} \\
17.5]\end{array}$ & $\begin{array}{c}10 / 26 / 2010 \\
15: 00\end{array}$ & $\begin{array}{c}{[-93.1} \\
20.4]\end{array}$ & 80 & $\begin{array}{c}10 / 25 / 2010 \\
3: 00\end{array}$ & $\begin{array}{c}{[-88.6,} \\
17.3]\end{array}$ \\
\hline al012016 & Hurricane Alex & $\begin{array}{c}1 / 13 / 2016 \\
21: 00\end{array}$ & $\begin{array}{c}{[-30.8} \\
27.1]\end{array}$ & $\begin{array}{c}1 / 15 / 2016 \\
21: 00\end{array}$ & $\begin{array}{c}{[-27.8,} \\
43.0]\end{array}$ & 75 & $\begin{array}{c}1 / 14 / 2016 \\
21: 00\end{array}$ & $\begin{array}{c}{[-27.8,} \\
33.6]\end{array}$ \\
\hline al042014 & Hurricane Cristobal & $\begin{array}{c}8 / 23 / 2014 \\
21: 00\end{array}$ & $\begin{array}{c}{[-72.3,} \\
21.8]\end{array}$ & $\begin{array}{c}8 / 29 / 2014 \\
15: 00\end{array}$ & $\begin{array}{c}{[-47.1} \\
45.5]\end{array}$ & 75 & 8/29/2014 3:00 & $\begin{array}{c}{[-56.6,} \\
40.2]\end{array}$ \\
\hline al052012 & Hurricane Ernesto & $\begin{array}{c}8 / 1 / 2012 \\
21: 00\end{array}$ & $\begin{array}{c}{[-49.0,} \\
12.2]\end{array}$ & $\begin{array}{c}8 / 10 / 2012 \\
15: 00\end{array}$ & $\begin{array}{c}{[-99.2} \\
18.0]\end{array}$ & 75 & 8/8/2012 3:00 & $\begin{array}{c}{[-87.7,} \\
18.7]\end{array}$ \\
\hline al062015 & Hurricane Fred & $\begin{array}{c}8 / 30 / 2015 \\
5: 30\end{array}$ & $\begin{array}{c}{[-18.4,} \\
12.1]\end{array}$ & $\begin{array}{c}9 / 6 / 2015 \\
21: 00\end{array}$ & $\begin{array}{c}{[-42.5,} \\
26.5]\end{array}$ & 75 & $\begin{array}{c}8 / 31 / 2015 \\
15: 00\end{array}$ & $\begin{array}{c}{[-23.7,} \\
16.4]\end{array}$ \\
\hline al072017 & Hurricane Franklin & $\begin{array}{c}8 / 6 / 2017 \\
21: 00\end{array}$ & $\begin{array}{c}{[-82.0,} \\
15.6]\end{array}$ & $\begin{array}{c}8 / 10 / 2017 \\
15: 00\end{array}$ & $\begin{array}{c}{[-99.2} \\
19.7]\end{array}$ & 75 & 8/10/2017 3:00 & $\begin{array}{c}{[-96.1} \\
20.2]\end{array}$ \\
\hline al092013 & Hurricane Humberto & $\begin{array}{c}9 / 8 / 2013 \\
21: 00\end{array}$ & $\begin{array}{c}{[-20.7} \\
13.1]\end{array}$ & $\begin{array}{c}9 / 19 / 2013 \\
21: 00\end{array}$ & $\begin{array}{c}{[-42.0,} \\
33.6]\end{array}$ & 75 & 9/12/2013 9:00 & $\begin{array}{c}{[-28.7,} \\
20.5]\end{array}$ \\
\hline
\end{tabular}




\begin{tabular}{|c|c|c|c|c|c|c|c|c|}
\hline al102013 & Hurricane Ingrid & $\begin{array}{c}9 / 12 / 2013 \\
21: 00\end{array}$ & $\begin{array}{c}{[-93.6} \\
19.7]\end{array}$ & $\begin{array}{c}9 / 17 / 2013 \\
9: 00\end{array}$ & $\begin{array}{c}{[-99.9} \\
23.7]\end{array}$ & 75 & 9/15/2013 9:00 & $\begin{array}{c}{[-95.4,} \\
22.4]\end{array}$ \\
\hline al172010 & Hurricane Otto & $\begin{array}{c}10 / 6 / 2010 \\
9: 00\end{array}$ & $\begin{array}{c}{[-67.0,} \\
22.2]\end{array}$ & $\begin{array}{c}10 / 10 / 2010 \\
15: 00\end{array}$ & $\begin{array}{c}{[-41.1} \\
37.3]\end{array}$ & 75 & 10/9/2010 9:00 & $\begin{array}{c}{[-58.5,} \\
29.2]\end{array}$ \\
\hline al022018 & Hurricane Beryl & $\begin{array}{c}7 / 5 / 2018 \\
15: 00\end{array}$ & $\begin{array}{c}{[-41.4,} \\
10.2]\end{array}$ & $\begin{array}{c}7 / 16 / 2018 \\
3: 00\end{array}$ & $\begin{array}{c}{[-62.4,} \\
38.6]\end{array}$ & 70 & 7/7/2018 0:00 & $\begin{array}{c}{[-48.3,} \\
10.6]\end{array}$ \\
\hline al032014 & Hurricane Bertha & 8/1/2014 3:00 & $\begin{array}{c}{[-55.5,} \\
12.3]\end{array}$ & $\begin{array}{c}8 / 6 / 2014 \\
15: 00\end{array}$ & $\begin{array}{c}{[-62.7} \\
40.2]\end{array}$ & 70 & 8/4/2014 15:00 & $\begin{array}{c}{[-73.6,} \\
27.6]\end{array}$ \\
\hline al052016 & Hurricane Earl & $\begin{array}{c}8 / 2 / 2016 \\
16: 00\end{array}$ & $\begin{array}{c}{[-80.2} \\
16.3]\end{array}$ & $\begin{array}{c}8 / 6 / 2016 \\
15: 00\end{array}$ & $\begin{array}{c}{[-98.0,} \\
19.0]\end{array}$ & 70 & 8/4/2016 6:00 & $\begin{array}{c}{[-88.3,} \\
17.4]\end{array}$ \\
\hline al082008 & Hurricane Hanna & $\begin{array}{c}8 / 28 / 2008 \\
9: 00\end{array}$ & $\begin{array}{c}{[-57.9,} \\
19.8]\end{array}$ & 9/7/2008 9:00 & $\begin{array}{c}{[-70.0,} \\
42.6]\end{array}$ & 70 & $9 / 2 / 20083: 00$ & $\begin{array}{c}{[-72.5,} \\
21.8]\end{array}$ \\
\hline al092012 & Hurricane Isaac & $\begin{array}{c}8 / 21 / 2012 \\
9: 00\end{array}$ & $\begin{array}{c}{[-51.2} \\
15.2]\end{array}$ & $\begin{array}{c}8 / 30 / 2012 \\
21: 00\end{array}$ & $\begin{array}{c}{[-92.6,} \\
32.7]\end{array}$ & 70 & 8/29/2012 5:00 & $\begin{array}{c}{[-90.0,} \\
29.0]\end{array}$ \\
\hline al092016 & Hurricane Hermine & $\begin{array}{c}8 / 28 / 2016 \\
21: 00\end{array}$ & $\begin{array}{c}{[-81.7,} \\
23.7]\end{array}$ & $\begin{array}{c}9 / 6 / 2016 \\
18: 00\end{array}$ & $\begin{array}{c}{[-72.3} \\
39.4]\end{array}$ & 70 & $9 / 2 / 20163: 00$ & $\begin{array}{c}{[-84.3,} \\
29.7]\end{array}$ \\
\hline al112008 & Hurricane Kyle & $\begin{array}{c}9 / 25 / 2008 \\
21: 00\end{array}$ & $\begin{array}{c}{[-68.3,} \\
23.5]\end{array}$ & $\begin{array}{c}9 / 29 / 2008 \\
3: 00\end{array}$ & $\begin{array}{c}{[-65.9,} \\
44.8]\end{array}$ & 70 & $\begin{array}{c}9 / 28 / 2008 \\
15: 00\end{array}$ & $\begin{array}{c}{[-67.7,} \\
40.4]\end{array}$ \\
\hline al142010 & Hurricane Lisa & $\begin{array}{c}9 / 21 / 2010 \\
3: 00\end{array}$ & $\begin{array}{c}{[-31.9} \\
17.1]\end{array}$ & $\begin{array}{c}9 / 26 / 2010 \\
21: 00\end{array}$ & $\begin{array}{c}{[-29.4} \\
26.1]\end{array}$ & 70 & 9/25/2010 3:00 & $\begin{array}{c}{[-27.9,} \\
20.7]\end{array}$ \\
\hline al142011 & Hurricane Maria & $\begin{array}{c}9 / 6 / 2011 \\
21: 00\end{array}$ & $\begin{array}{c}{[-37.0,} \\
11.8]\end{array}$ & $\begin{array}{c}9 / 16 / 2011 \\
21: 00\end{array}$ & $\begin{array}{c}{[-51.5,} \\
48.5]\end{array}$ & 70 & 9/16/2011 9:00 & $\begin{array}{c}{[-60.3,} \\
41.7]\end{array}$ \\
\hline al182019 & Hurricane Pablo & $\begin{array}{c}10 / 25 / 2019 \\
21: 00\end{array}$ & $\begin{array}{c}{[-32.2,} \\
35.8]\end{array}$ & $\begin{array}{c}10 / 28 / 2019 \\
15: 00\end{array}$ & $\begin{array}{c}{[-17.7} \\
46.8]\end{array}$ & 70 & $\begin{array}{c}10 / 27 / 2019 \\
21: 00\end{array}$ & $\begin{array}{c}{[-17.2,} \\
44.7]\end{array}$ \\
\hline al022019 & Hurricane Barry & $\begin{array}{c}7 / 10 / 2019 \\
15: 00\end{array}$ & $\begin{array}{c}{[-86.4,} \\
28.5]\end{array}$ & $\begin{array}{c}7 / 14 / 2019 \\
21: 00\end{array}$ & $\begin{array}{c}{[-93.6} \\
32.8]\end{array}$ & 65 & $\begin{array}{c}7 / 13 / 2019 \\
15: 00\end{array}$ & $\begin{array}{c}{[-92.0,} \\
29.6]\end{array}$ \\
\hline al032012 & Hurricane Chris & $\begin{array}{c}6 / 19 / 2012 \\
21: 00\end{array}$ & $\begin{array}{c}{[-57.7} \\
39.3]\end{array}$ & $\begin{array}{c}6 / 22 / 2012 \\
15: 00\end{array}$ & $\begin{array}{c}{[-46.9,} \\
44.6]\end{array}$ & 65 & $\begin{array}{c}6 / 21 / 2012 \\
15: 00\end{array}$ & $\begin{array}{c}{[-43.2,} \\
41.1]\end{array}$ \\
\hline al072014 & Hurricane Fay & $\begin{array}{c}10 / 10 / 2014 \\
15: 00\end{array}$ & $\begin{array}{c}{[-63.7,} \\
23.8]\end{array}$ & $\begin{array}{c}10 / 13 / 2014 \\
21: 00\end{array}$ & $\begin{array}{c}{[-49.9} \\
33.7]\end{array}$ & 65 & $\begin{array}{c}10 / 12 / 2014 \\
21: 00\end{array}$ & $\begin{array}{c}{[-60.7,} \\
34.7]\end{array}$ \\
\hline al092018 & Hurricane Isaac & $\begin{array}{c}9 / 7 / 2018 \\
21: 00\end{array}$ & $\begin{array}{c}{[-34.9} \\
13.6]\end{array}$ & $\begin{array}{c}9 / 15 / 2018 \\
9: 00\end{array}$ & $\begin{array}{c}{[-70.8,} \\
14.8]\end{array}$ & 65 & $\begin{array}{c}9 / 10 / 2018 \\
15: 00\end{array}$ & $\begin{array}{c}{[-43.9,} \\
14.7]\end{array}$ \\
\hline
\end{tabular}




\begin{tabular}{|c|c|c|c|c|c|c|c|c|}
\hline al122012 & Hurricane Leslie & $\begin{array}{c}8 / 30 / 2012 \\
15: 00\end{array}$ & $\begin{array}{c}{[-43.4} \\
14.1]\end{array}$ & $\begin{array}{c}9 / 11 / 2012 \\
15: 00\end{array}$ & $\begin{array}{c}{[-53.6,} \\
49.4]\end{array}$ & 65 & 9/7/2012 0:00 & $\begin{array}{c}{[-62.2,} \\
26.5]\end{array}$ \\
\hline al122015 & Hurricane Kate & $\begin{array}{c}11 / 9 / 2015 \\
3: 00\end{array}$ & $\begin{array}{c}{[-73.0,} \\
23.0]\end{array}$ & $\begin{array}{c}11 / 12 / 2015 \\
9: 00\end{array}$ & $\begin{array}{c}{[-50.8} \\
40.7]\end{array}$ & 65 & $\begin{array}{c}11 / 11 / 2015 \\
15: 00\end{array}$ & $\begin{array}{c}{[-60.5,} \\
36.8]\end{array}$ \\
\hline al202010 & Hurricane Shary & $\begin{array}{c}10 / 29 / 2010 \\
3: 00\end{array}$ & $\begin{array}{c}{[-63.7} \\
27.3]\end{array}$ & $\begin{array}{c}10 / 30 / 2010 \\
21: 00\end{array}$ & $\begin{array}{c}{[-50.9} \\
39.2]\end{array}$ & 65 & $\begin{array}{c}10 / 30 / 2010 \\
15: 00\end{array}$ & $\begin{array}{c}{[-55.6,} \\
35.9]\end{array}$ \\
\hline al022012 & Tropical Storm Beryl & $\begin{array}{c}5 / 26 / 2012 \\
3: 00\end{array}$ & $\begin{array}{c}{[-74.8,} \\
32.5]\end{array}$ & $\begin{array}{c}5 / 30 / 2012 \\
21: 00\end{array}$ & $\begin{array}{c}{[-76.1} \\
34.9]\end{array}$ & 61 & $5 / 28 / 20120: 00$ & $\begin{array}{c}{[-80.5,} \\
30.1]\end{array}$ \\
\hline al152011 & Hurricane Nate & $\begin{array}{c}9 / 7 / 2011 \\
21: 00\end{array}$ & $\begin{array}{c}{[-92.4} \\
20.2]\end{array}$ & $\begin{array}{c}9 / 12 / 2011 \\
3: 00\end{array}$ & $\begin{array}{c}{[-98.4} \\
20.5]\end{array}$ & 61 & 9/9/2011 6:00 & $\begin{array}{c}{[-92.2,} \\
19.7]\end{array}$ \\
\hline al072018 & $\begin{array}{c}\text { Tropical Storm } \\
\text { Gordon }\end{array}$ & $\begin{array}{c}9 / 2 / 2018 \\
21: 00\end{array}$ & $\begin{array}{c}{[-77.3} \\
22.7]\end{array}$ & $\begin{array}{c}9 / 5 / 2018 \\
15: 00\end{array}$ & $\begin{array}{c}{[-90.2} \\
32.3]\end{array}$ & 60 & 9/5/2018 0:00 & $\begin{array}{c}{[-87.9,} \\
29.7]\end{array}$ \\
\hline al092009 & Tropical Storm Grace & $\begin{array}{c}10 / 5 / 2009 \\
3: 00\end{array}$ & $\begin{array}{c}{[-20.3,} \\
41.2]\end{array}$ & $\begin{array}{c}10 / 6 / 2009 \\
3: 00\end{array}$ & $\begin{array}{c}{[-13.4} \\
49.7]\end{array}$ & 60 & 10/5/2009 9:00 & $\begin{array}{c}{[-18.0,} \\
43.0]\end{array}$ \\
\hline al122016 & Tropical Storm Karl & $\begin{array}{c}9 / 14 / 2016 \\
15: 00\end{array}$ & $\begin{array}{c}{[-25.0,} \\
17.0]\end{array}$ & $\begin{array}{c}9 / 25 / 2016 \\
15: 00\end{array}$ & $\begin{array}{c}{[-47.9,} \\
39.9]\end{array}$ & 60 & $\begin{array}{c}9 / 25 / 2016 \\
15: 00\end{array}$ & $\begin{array}{c}{[-47.9,} \\
39.9]\end{array}$ \\
\hline al012011 & $\begin{array}{c}\text { Tropical Storm } \\
\text { Arlene }\end{array}$ & $\begin{array}{c}6 / 29 / 2011 \\
0: 00\end{array}$ & $\begin{array}{c}{[-93.7} \\
21.2]\end{array}$ & 7/1/2011 3:00 & $\begin{array}{c}{[-99.1} \\
20.9]\end{array}$ & 56 & $\begin{array}{c}6 / 30 / 2011 \\
12: 00\end{array}$ & $\begin{array}{c}{[-97.5,} \\
21.6]\end{array}$ \\
\hline al032013 & $\begin{array}{c}\text { Tropical Storm } \\
\text { Chantal }\end{array}$ & 7/8/2013 3:00 & $\begin{array}{c}{[-47.2} \\
9.8]\end{array}$ & $\begin{array}{c}7 / 10 / 2013 \\
21: 00\end{array}$ & $\begin{array}{c}{[-73.7} \\
16.5]\end{array}$ & 56 & $7 / 10 / 2013$ 0:00 & $\begin{array}{c}{[-64.9,} \\
15.4]\end{array}$ \\
\hline al102010 & $\begin{array}{c}\text { Tropical Storm } \\
\text { Hermine }\end{array}$ & 9/6/2010 3:00 & $\begin{array}{c}{[-95.2} \\
20.7]\end{array}$ & 9/8/2010 3:00 & $\begin{array}{c}{[-99.5,} \\
31.6]\end{array}$ & 56 & 9/7/2010 0:00 & $\begin{array}{c}{[-97.1,} \\
24.8]\end{array}$ \\
\hline al122013 & Tropical Storm Karen & $\begin{array}{c}10 / 3 / 2013 \\
13: 00\end{array}$ & $\begin{array}{c}{[-87.6,} \\
22.0]\end{array}$ & $\begin{array}{c}10 / 6 / 2013 \\
15: 00\end{array}$ & $\begin{array}{c}{[-89.9} \\
28.1]\end{array}$ & 56 & 10/4/2013 0:00 & $\begin{array}{c}{[-88.9,} \\
23.8]\end{array}$ \\
\hline al192011 & Tropical Storm Sean & $\begin{array}{c}11 / 8 / 2011 \\
9: 00\end{array}$ & $\begin{array}{c}{[-69.4,} \\
27.2]\end{array}$ & $\begin{array}{c}11 / 12 / 2011 \\
3: 00\end{array}$ & $\begin{array}{c}{[-57.7} \\
36.7]\end{array}$ & 56 & $\begin{array}{c}11 / 10 / 2011 \\
18: 00\end{array}$ & $\begin{array}{c}{[-70.0,} \\
30.5]\end{array}$ \\
\hline al012013 & $\begin{array}{c}\text { Tropical Storm } \\
\text { Andrea }\end{array}$ & $\begin{array}{c}6 / 5 / 2013 \\
22: 00\end{array}$ & $\begin{array}{c}{[-86.5,} \\
25.3]\end{array}$ & $\begin{array}{c}6 / 8 / 2013 \\
15: 00\end{array}$ & $\begin{array}{c}{[-68.8,} \\
43.6]\end{array}$ & 55 & 6/6/2013 21:00 & $\begin{array}{c}{[-83.4,} \\
29.5]\end{array}$ \\
\hline al012018 & $\begin{array}{l}\text { Tropical Storm } \\
\text { Alberto }\end{array}$ & $\begin{array}{c}5 / 25 / 2018 \\
15: 00\end{array}$ & $\begin{array}{c}{[-86.8,} \\
19.7]\end{array}$ & $\begin{array}{c}5 / 29 / 2018 \\
9: 00\end{array}$ & $\begin{array}{c}{[-86.8,} \\
32.3]\end{array}$ & 55 & 5/28/2018 6:00 & $\begin{array}{c}{[-86.1,} \\
28.6]\end{array}$ \\
\hline al022011 & Tropical Storm Bret & $\begin{array}{c}7 / 17 / 2011 \\
21: 00\end{array}$ & $\begin{array}{c}{[-78.2} \\
27.5]\end{array}$ & $\begin{array}{c}7 / 22 / 2011 \\
15: 00\end{array}$ & $\begin{array}{c}{[-64.2} \\
37.7]\end{array}$ & 55 & $\begin{array}{c}7 / 18 / 2011 \\
21: 00\end{array}$ & $\begin{array}{c}{[-77.0,} \\
28.3]\end{array}$ \\
\hline
\end{tabular}




\begin{tabular}{|c|c|c|c|c|c|c|c|c|}
\hline al032008 & $\begin{array}{c}\text { Tropical Storm } \\
\text { Cristobal }\end{array}$ & $\begin{array}{c}7 / 19 / 2008 \\
3: 00\end{array}$ & $\begin{array}{c}{[-79.6,} \\
31.9]\end{array}$ & $\begin{array}{c}7 / 23 / 2008 \\
9: 00\end{array}$ & $\begin{array}{c}{[-55.9} \\
44.7]\end{array}$ & 55 & $\begin{array}{c}7 / 21 / 2008 \\
21: 00\end{array}$ & $\begin{array}{c}{[-71.4,} \\
37.1]\end{array}$ \\
\hline al052008 & $\begin{array}{c}\text { Tropical Storm } \\
\text { Edouard }\end{array}$ & $\begin{array}{c}8 / 3 / 2008 \\
21: 00\end{array}$ & $\begin{array}{c}{[-88.1} \\
28.2]\end{array}$ & $\begin{array}{c}8 / 5 / 2008 \\
21: 00\end{array}$ & $\begin{array}{c}{[-95.2} \\
30.2]\end{array}$ & 55 & $8 / 5 / 200812: 00$ & $\begin{array}{c}{[-94.2,} \\
29.6]\end{array}$ \\
\hline al062008 & Tropical Storm Fay & $\begin{array}{c}8 / 15 / 2008 \\
21: 00\end{array}$ & $\begin{array}{c}{[-69.4} \\
18.5]\end{array}$ & $\begin{array}{c}8 / 24 / 2008 \\
3: 00\end{array}$ & $\begin{array}{c}{[-87.1} \\
30.9]\end{array}$ & 55 & $\begin{array}{c}8 / 19 / 2008 \\
21: 00\end{array}$ & $\begin{array}{c}{[-81.0,} \\
27.3]\end{array}$ \\
\hline al082019 & $\begin{array}{c}\text { Tropical Storm } \\
\text { Gabrielle }\end{array}$ & $\begin{array}{c}9 / 3 / 2019 \\
21: 00\end{array}$ & $\begin{array}{c}{[-32.3} \\
19.0]\end{array}$ & $\begin{array}{c}9 / 10 / 2019 \\
15: 00\end{array}$ & $\begin{array}{c}{[-37.8} \\
43.9]\end{array}$ & 55 & 9/9/2019 3:00 & $\begin{array}{c}{[-49.3,} \\
36.5]\end{array}$ \\
\hline al102008 & $\begin{array}{l}\text { Tropical Storm } \\
\text { Josephine }\end{array}$ & 9/2/2008 9:00 & $\begin{array}{c}{[-23.9} \\
12.4]\end{array}$ & 9/6/2008 9:00 & $\begin{array}{c}{[-36.9} \\
16.8]\end{array}$ & 55 & $9 / 3 / 2008$ 15:00 & $\begin{array}{c}{[-28.8,} \\
13.8]\end{array}$ \\
\hline al102016 & Tropical Storm Ian & $\begin{array}{c}9 / 12 / 2016 \\
15: 00\end{array}$ & $\begin{array}{c}{[-50.4,} \\
21.8]\end{array}$ & $\begin{array}{c}9 / 16 / 2016 \\
15: 00\end{array}$ & $\begin{array}{c}{[-36.2} \\
48.8]\end{array}$ & 55 & $\begin{array}{c}9 / 16 / 2016 \\
15: 00\end{array}$ & $\begin{array}{c}{[-36.2,} \\
48.8]\end{array}$ \\
\hline al132008 & $\begin{array}{c}\text { Tropical Storm } \\
\text { Marco }\end{array}$ & $\begin{array}{c}10 / 6 / 2008 \\
15: 00\end{array}$ & $\begin{array}{c}{[-94.1} \\
19.0]\end{array}$ & $\begin{array}{c}10 / 8 / 2008 \\
3: 00\end{array}$ & $\begin{array}{c}{[-97.5} \\
20.0]\end{array}$ & 55 & 10/7/2008 6:00 & $\begin{array}{c}{[-95.7,} \\
19.9]\end{array}$ \\
\hline al142013 & $\begin{array}{c}\text { Tropical Storm } \\
\text { Melissa }\end{array}$ & $\begin{array}{c}11 / 18 / 2013 \\
15: 00\end{array}$ & $\begin{array}{c}{[-53.6} \\
29.3]\end{array}$ & $\begin{array}{c}11 / 22 / 2013 \\
3: 00\end{array}$ & $\begin{array}{c}{[-29.0} \\
41.5]\end{array}$ & 55 & $\begin{array}{c}11 / 19 / 2013 \\
21: 00\end{array}$ & $\begin{array}{c}{[-53.7,} \\
33.0]\end{array}$ \\
\hline al142019 & $\begin{array}{c}\text { Tropical Storm } \\
\text { Melissa }\end{array}$ & $\begin{array}{c}10 / 11 / 2019 \\
15: 00\end{array}$ & $\begin{array}{c}{[-69.6,} \\
38.5]\end{array}$ & $\begin{array}{c}10 / 14 / 2019 \\
15: 00\end{array}$ & $\begin{array}{c}{[-51.4,} \\
41.0]\end{array}$ & 55 & $\begin{array}{c}10 / 11 / 2019 \\
15: 00\end{array}$ & $\begin{array}{c}{[-69.6,} \\
38.5]\end{array}$ \\
\hline al152018 & $\begin{array}{c}\text { Tropical Storm } \\
\text { Nadine }\end{array}$ & $\begin{array}{c}10 / 9 / 2018 \\
10: 00\end{array}$ & $\begin{array}{c}{[-29.7} \\
10.3]\end{array}$ & $\begin{array}{c}10 / 13 / 2018 \\
3: 00\end{array}$ & $\begin{array}{c}{[-39.5,} \\
16.0]\end{array}$ & 55 & $\begin{array}{c}10 / 11 / 2018 \\
3: 00\end{array}$ & $\begin{array}{c}{[-32.7,} \\
13.6]\end{array}$ \\
\hline al202019 & $\begin{array}{l}\text { Tropical Storm } \\
\text { Sebastien }\end{array}$ & $\begin{array}{c}11 / 19 / 2019 \\
15: 00\end{array}$ & $\begin{array}{c}{[-58.7} \\
20.1]\end{array}$ & $\begin{array}{c}11 / 25 / 2019 \\
3: 00\end{array}$ & $\begin{array}{c}{[-28.9,} \\
41.0]\end{array}$ & 55 & $\begin{array}{c}11 / 24 / 2019 \\
3: 00\end{array}$ & $\begin{array}{c}{[-43.1,} \\
34.4]\end{array}$ \\
\hline al012015 & Tropical Storm Ana & 5/8/2015 3:00 & $\begin{array}{c}{[-77.6,} \\
31.5]\end{array}$ & $\begin{array}{c}\text { 5/10/2015 } \\
21: 00\end{array}$ & $\begin{array}{c}{[-78.6,} \\
34.4]\end{array}$ & 52 & 5/9/2015 18:00 & $\begin{array}{c}{[-78.1,} \\
32.7]\end{array}$ \\
\hline al022015 & Tropical Storm Bill & $\begin{array}{c}6 / 16 / 2015 \\
3: 00\end{array}$ & $\begin{array}{c}{[-94.2} \\
27.1]\end{array}$ & $\begin{array}{c}\text { 6/17/2015 } \\
9: 00\end{array}$ & $\begin{array}{c}{[-97.1} \\
31.0]\end{array}$ & 52 & $\begin{array}{c}6 / 16 / 2015 \\
18: 00\end{array}$ & $\begin{array}{c}{[-96.7,} \\
28.2]\end{array}$ \\
\hline al032016 & Tropical Storm Colin & $\begin{array}{c}6 / 5 / 2016 \\
15: 00\end{array}$ & $\begin{array}{c}{[-88.1} \\
21.9]\end{array}$ & $\begin{array}{c}6 / 7 / 2016 \\
21: 00\end{array}$ & $\begin{array}{c}{[-72.2} \\
36.5]\end{array}$ & 52 & 6/7/2016 18:00 & $\begin{array}{c}{[-74.7,} \\
35.3]\end{array}$ \\
\hline al042012 & $\begin{array}{c}\text { Tropical Storm } \\
\text { Debby }\end{array}$ & $\begin{array}{c}6 / 23 / 2012 \\
21: 00\end{array}$ & $\begin{array}{c}{[-87.6,} \\
26.2]\end{array}$ & $\begin{array}{c}6 / 27 / 2012 \\
21: 00\end{array}$ & $\begin{array}{c}{[-78.3,} \\
29.5]\end{array}$ & 52 & $6 / 25 / 20120: 00$ & $\begin{array}{c}{[-86.0,} \\
28.3]\end{array}$ \\
\hline al062009 & Tropical Storm Erika & $\begin{array}{c}9 / 1 / 2009 \\
21: 00\end{array}$ & $\begin{array}{c}{[-57.3,} \\
17.2]\end{array}$ & 9/4/2009 3:00 & $\begin{array}{c}{[-65.6,} \\
16.8]\end{array}$ & 52 & 9/2/2009 6:00 & $\begin{array}{c}{[-58.7,} \\
17.0]\end{array}$ \\
\hline
\end{tabular}




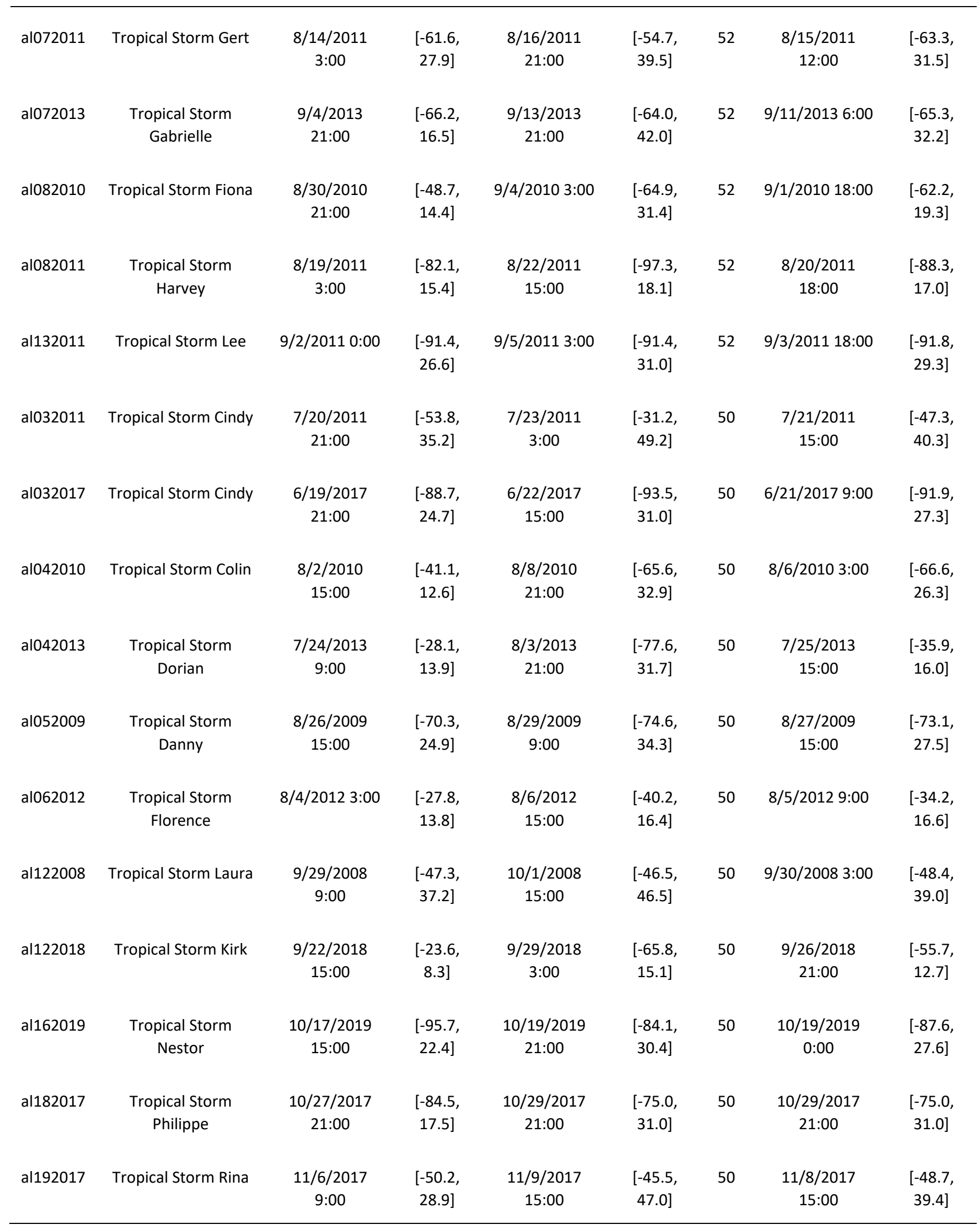




\begin{tabular}{|c|c|c|c|c|c|c|c|c|}
\hline al012012 & $\begin{array}{c}\text { Tropical Storm } \\
\text { Alberto }\end{array}$ & $\begin{array}{c}5 / 19 / 2012 \\
21: 00\end{array}$ & $\begin{array}{c}{[-77.7} \\
32.2]\end{array}$ & $\begin{array}{c}5 / 22 / 2012 \\
15: 00\end{array}$ & $\begin{array}{c}{[-74.0,} \\
33.1]\end{array}$ & 45 & 5/20/2012 9:00 & $\begin{array}{c}{[-78.9,} \\
31.7]\end{array}$ \\
\hline al012017 & $\begin{array}{c}\text { Tropical Storm } \\
\text { Arlene }\end{array}$ & $\begin{array}{c}4 / 19 / 2017 \\
15: 00\end{array}$ & $\begin{array}{c}{[-40.9,} \\
31.9]\end{array}$ & $\begin{array}{c}4 / 21 / 2017 \\
15: 00\end{array}$ & $\begin{array}{c}{[-49.9} \\
39.3]\end{array}$ & 45 & 4/21/2017 9:00 & $\begin{array}{c}{[-48.0,} \\
40.0]\end{array}$ \\
\hline al032015 & $\begin{array}{l}\text { Tropical Storm } \\
\text { Claudette }\end{array}$ & $\begin{array}{c}7 / 13 / 2015 \\
17: 00\end{array}$ & $\begin{array}{c}{[-68.1} \\
37.4]\end{array}$ & $\begin{array}{c}7 / 15 / 2015 \\
3: 00\end{array}$ & $\begin{array}{c}{[-57.8,} \\
43.8]\end{array}$ & 45 & $\begin{array}{c}\text { 7/13/2015 } \\
21: 00\end{array}$ & $\begin{array}{c}{[-66.9} \\
38.1]\end{array}$ \\
\hline al042009 & $\begin{array}{l}\text { Tropical Storm } \\
\text { Claudette }\end{array}$ & $\begin{array}{c}8 / 16 / 2009 \\
9: 00\end{array}$ & $\begin{array}{c}{[-83.9} \\
27.7]\end{array}$ & $\begin{array}{c}8 / 17 / 2009 \\
12: 00\end{array}$ & $\begin{array}{c}{[-87.2} \\
31.3]\end{array}$ & 45 & $\begin{array}{c}8 / 16 / 2009 \\
21: 00\end{array}$ & $\begin{array}{c}{[-85.6,} \\
29.5]\end{array}$ \\
\hline al042011 & Tropical Storm Don & $\begin{array}{c}7 / 27 / 2011 \\
21: 00\end{array}$ & $\begin{array}{c}{[-87.0,} \\
22.2]\end{array}$ & $\begin{array}{c}7 / 30 / 2011 \\
9: 00\end{array}$ & $\begin{array}{c}{[-98.8,} \\
27.9]\end{array}$ & 45 & $\begin{array}{c}7 / 29 / 2011 \\
15: 00\end{array}$ & $\begin{array}{c}{[-94.9,} \\
26.2]\end{array}$ \\
\hline al042018 & $\begin{array}{c}\text { Tropical Storm } \\
\text { Debby }\end{array}$ & $\begin{array}{c}8 / 7 / 2018 \\
15: 00\end{array}$ & $\begin{array}{c}{[-48.5,} \\
38.9]\end{array}$ & $\begin{array}{c}8 / 9 / 2018 \\
21: 00\end{array}$ & $\begin{array}{c}{[-40.5,} \\
45.5]\end{array}$ & 45 & 8/9/2018 3:00 & $\begin{array}{c}{[-46.5,} \\
42.8]\end{array}$ \\
\hline al052011 & Tropical Storm Emily & $\begin{array}{c}8 / 1 / 2011 \\
23: 30\end{array}$ & $\begin{array}{c}{[-62.0} \\
15.2]\end{array}$ & $\begin{array}{c}8 / 7 / 2011 \\
21: 00\end{array}$ & $\begin{array}{c}{[-74.1} \\
31.1]\end{array}$ & 45 & $8 / 3 / 201121: 00$ & $\begin{array}{c}{[-71.0,} \\
16.9]\end{array}$ \\
\hline al052014 & Tropical Storm Dolly & $\begin{array}{c}9 / 1 / 2014 \\
21: 00\end{array}$ & $\begin{array}{c}{[-93.6} \\
20.1]\end{array}$ & $\begin{array}{c}9 / 3 / 2014 \\
15: 00\end{array}$ & $\begin{array}{c}{[-99.2} \\
21.7]\end{array}$ & 45 & 9/2/2014 15:00 & $\begin{array}{c}{[-96.5,} \\
23.4]\end{array}$ \\
\hline al052015 & Tropical Storm Erika & $\begin{array}{c}8 / 25 / 2015 \\
3: 00\end{array}$ & $\begin{array}{c}{[-47.7,} \\
14.4]\end{array}$ & $\begin{array}{c}8 / 29 / 2015 \\
13: 30\end{array}$ & $\begin{array}{c}{[-75.9} \\
21.5]\end{array}$ & 45 & 8/28/2015 9:00 & $\begin{array}{c}{[-67.7,} \\
17.7]\end{array}$ \\
\hline al052017 & Tropical Storm Don & $\begin{array}{c}7 / 17 / 2017 \\
21: 00\end{array}$ & $\begin{array}{c}{[-52.6} \\
11.2]\end{array}$ & $\begin{array}{c}7 / 19 / 2017 \\
3: 00\end{array}$ & $\begin{array}{c}{[-62.5,} \\
11.9]\end{array}$ & 45 & $\begin{array}{c}7 / 18 / 2017 \\
12: 00\end{array}$ & $\begin{array}{c}{[-57.0,} \\
11.4]\end{array}$ \\
\hline al062013 & $\begin{array}{l}\text { Tropical Storm } \\
\text { Fernand }\end{array}$ & $\begin{array}{c}8 / 25 / 2013 \\
21: 00\end{array}$ & $\begin{array}{c}{[-95.5} \\
19.5]\end{array}$ & $\begin{array}{c}8 / 26 / 2013 \\
21: 00\end{array}$ & $\begin{array}{c}{[-98.5,} \\
20.6]\end{array}$ & 45 & 8/26/2013 3:00 & $\begin{array}{c}{[-96.2,} \\
19.3]\end{array}$ \\
\hline al062016 & Tropical Storm Fiona & $\begin{array}{c}8 / 17 / 2016 \\
3: 00\end{array}$ & $\begin{array}{c}{[-34.1} \\
12.6]\end{array}$ & $\begin{array}{c}8 / 23 / 2016 \\
15: 00\end{array}$ & $\begin{array}{c}{[-64.5,} \\
26.1]\end{array}$ & 45 & 8/21/2016 3:00 & $\begin{array}{c}{[-50.1} \\
21.7]\end{array}$ \\
\hline al072015 & Tropical Storm Grace & $\begin{array}{c}9 / 5 / 2015 \\
15: 00\end{array}$ & $\begin{array}{c}{[-25.2} \\
12.3]\end{array}$ & $\begin{array}{c}9 / 9 / 2015 \\
15: 00\end{array}$ & $\begin{array}{c}{[-49.0,} \\
14.5]\end{array}$ & 45 & 9/7/2015 3:00 & $\begin{array}{c}{[-32.5,} \\
13.3]\end{array}$ \\
\hline al072019 & $\begin{array}{l}\text { Tropical Storm } \\
\text { Fernand }\end{array}$ & 9/3/2019 9:00 & $\begin{array}{c}{[-94.3} \\
23.5]\end{array}$ & 9/5/2019 3:00 & $\begin{array}{c}{[-99.0,} \\
25.0]\end{array}$ & 45 & 9/4/2019 6:00 & $\begin{array}{c}{[-96.7,} \\
23.0]\end{array}$ \\
\hline al102009 & Tropical Storm Henri & $\begin{array}{c}10 / 6 / 2009 \\
21: 00\end{array}$ & $\begin{array}{c}{[-54.0} \\
17.8]\end{array}$ & $\begin{array}{c}10 / 8 / 2009 \\
21: 00\end{array}$ & $\begin{array}{c}{[-62.2} \\
20.4]\end{array}$ & 45 & 10/7/2009 9:00 & $\begin{array}{c}{[-56.1} \\
18.1]\end{array}$ \\
\hline al102015 & Tropical Storm Ida & $\begin{array}{c}9 / 18 / 2015 \\
15: 00\end{array}$ & $\begin{array}{c}{[-36.7} \\
13.1]\end{array}$ & $\begin{array}{c}9 / 27 / 2015 \\
21: 00\end{array}$ & $\begin{array}{c}{[-48.7,} \\
24.5]\end{array}$ & 45 & $\begin{array}{c}9 / 21 / 2015 \\
15: 00\end{array}$ & $\begin{array}{c}{[-48.0,} \\
20.6]\end{array}$ \\
\hline
\end{tabular}




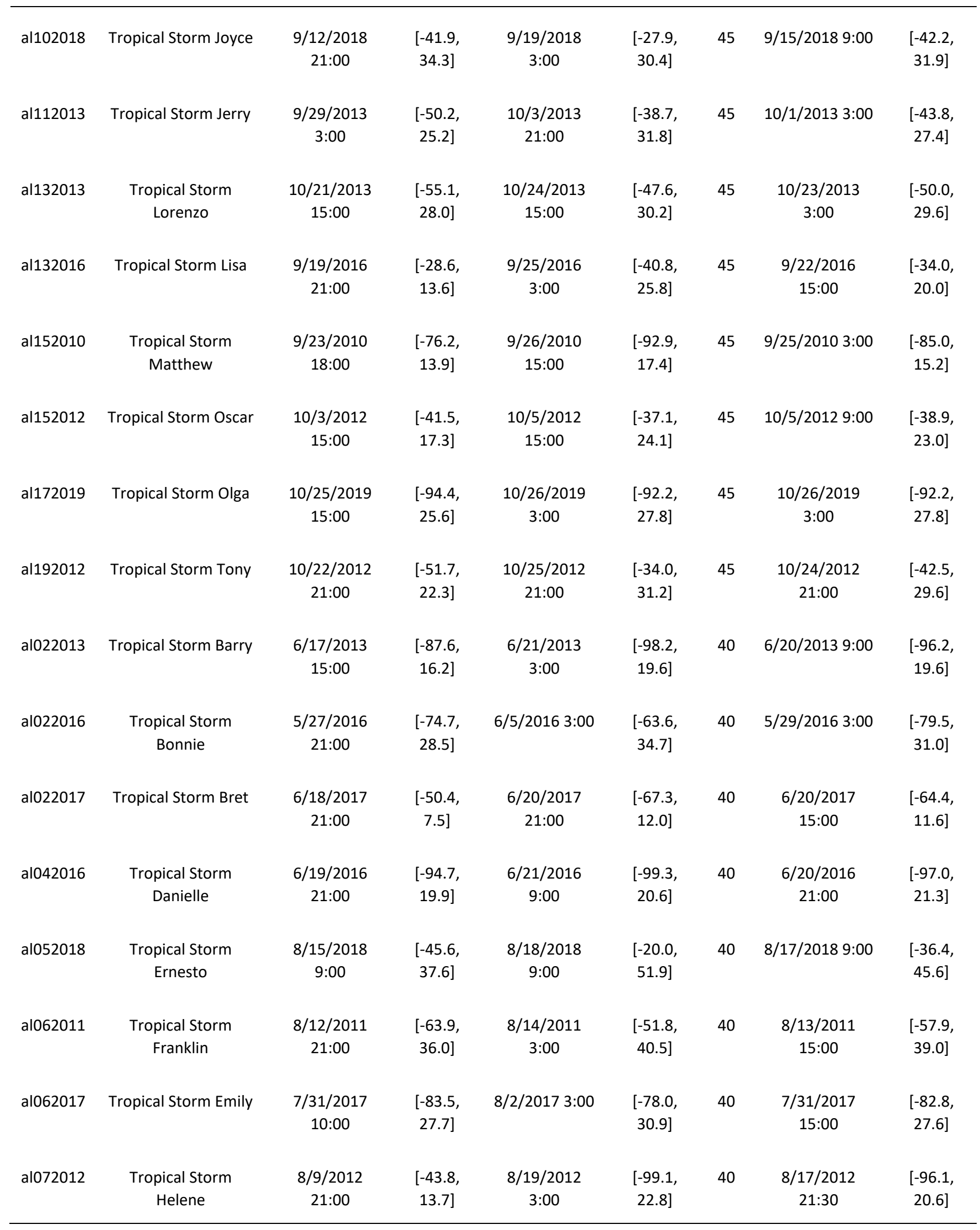




\begin{tabular}{|c|c|c|c|c|c|c|c|c|}
\hline al102017 & $\begin{array}{c}\text { Atmospheric System } \\
\text { Ten }\end{array}$ & $\begin{array}{c}8 / 27 / 2017 \\
21: 00\end{array}$ & $\begin{array}{c}{[-80.0} \\
31.2]\end{array}$ & $\begin{array}{c}8 / 29 / 2017 \\
21: 00\end{array}$ & $\begin{array}{c}{[-74.3,} \\
36.0]\end{array}$ & 40 & $\begin{array}{c}8 / 29 / 2017 \\
21: 00\end{array}$ & $\begin{array}{c}{[-74.3,} \\
36.0]\end{array}$ \\
\hline al112011 & Tropical Storm Jose & $\begin{array}{c}8 / 28 / 2011 \\
12: 00\end{array}$ & $\begin{array}{c}{[-65.7} \\
30.8]\end{array}$ & $\begin{array}{c}8 / 29 / 2011 \\
15: 00\end{array}$ & $\begin{array}{c}{[-63.1} \\
39.5]\end{array}$ & 40 & $\begin{array}{c}8 / 28 / 2011 \\
21: 00\end{array}$ & $\begin{array}{c}{[-65.7,} \\
33.2]\end{array}$ \\
\hline al122019 & Tropical Storm Karen & $\begin{array}{c}9 / 22 / 2019 \\
9: 00\end{array}$ & $\begin{array}{c}{[-60.2} \\
11.9]\end{array}$ & $\begin{array}{c}9 / 27 / 2019 \\
21: 00\end{array}$ & $\begin{array}{c}{[-58.5} \\
29.3]\end{array}$ & 40 & $9 / 25 / 20196: 00$ & $\begin{array}{c}{[-65.1,} \\
19.8]\end{array}$ \\
\hline al162012 & Tropical Storm Patty & $\begin{array}{c}10 / 11 / 2012 \\
15: 00\end{array}$ & $\begin{array}{c}{[-72.6} \\
25.4]\end{array}$ & $\begin{array}{c}10 / 13 / 2012 \\
15: 00\end{array}$ & $\begin{array}{c}{[-72.6} \\
24.8]\end{array}$ & 40 & $\begin{array}{c}10 / 12 / 2012 \\
3: 00\end{array}$ & $\begin{array}{c}{[-72.5,} \\
25.9]\end{array}$ \\
\hline al012008 & $\begin{array}{c}\text { Tropical Storm } \\
\text { Arthur }\end{array}$ & $\begin{array}{c}5 / 31 / 2008 \\
17: 00\end{array}$ & $\begin{array}{c}{[-88.5} \\
18.1]\end{array}$ & 6/2/2008 3:00 & $\begin{array}{c}{[-91.3} \\
17.4]\end{array}$ & 35 & 6/1/2008 3:00 & $\begin{array}{c}{[-89.5} \\
18.4]\end{array}$ \\
\hline al012019 & $\begin{array}{c}\text { Tropical Storm } \\
\text { Andrea }\end{array}$ & $\begin{array}{c}5 / 20 / 2019 \\
22: 00\end{array}$ & $\begin{array}{c}{[-68.7} \\
28.8]\end{array}$ & $\begin{array}{c}5 / 21 / 2019 \\
21: 00\end{array}$ & $\begin{array}{c}{[-68.3} \\
30.8]\end{array}$ & 35 & 5/21/2019 3:00 & $\begin{array}{c}{[-68.7,} \\
29.5]\end{array}$ \\
\hline al022009 & Tropical Storm Ana & $\begin{array}{c}8 / 11 / 2009 \\
10: 00\end{array}$ & $\begin{array}{c}{[-28.6} \\
14.4]\end{array}$ & $\begin{array}{c}8 / 17 / 2009 \\
21: 00\end{array}$ & $\begin{array}{c}{[-68.0} \\
17.5]\end{array}$ & 35 & $8 / 16 / 20096: 00$ & $\begin{array}{c}{[-52.5,} \\
14.5]\end{array}$ \\
\hline al042019 & $\begin{array}{c}\text { Tropical Storm } \\
\text { Chantal }\end{array}$ & $\begin{array}{c}8 / 21 / 2019 \\
3: 00\end{array}$ & $\begin{array}{c}{[-56.2} \\
40.2]\end{array}$ & $\begin{array}{c}8 / 24 / 2019 \\
3: 00\end{array}$ & $\begin{array}{c}{[-40.9,} \\
35.6]\end{array}$ & 35 & $\begin{array}{c}8 / 21 / 2019 \\
15: 00\end{array}$ & $\begin{array}{c}{[-51.6,} \\
40.2]\end{array}$ \\
\hline al052013 & Tropical Storm Erin & $\begin{array}{c}8 / 15 / 2013 \\
3: 00\end{array}$ & $\begin{array}{c}{[-23.5} \\
14.0]\end{array}$ & $\begin{array}{c}8 / 18 / 2013 \\
21: 00\end{array}$ & $\begin{array}{c}{[-39.8} \\
20.4]\end{array}$ & 35 & 8/16/2013 9:00 & $\begin{array}{c}{[-30.5} \\
16.3]\end{array}$ \\
\hline al062019 & Tropical Storm Erin & $\begin{array}{c}8 / 26 / 2019 \\
21: 00\end{array}$ & $\begin{array}{c}{[-72.5} \\
31.7]\end{array}$ & $\begin{array}{c}8 / 29 / 2019 \\
9: 00\end{array}$ & $\begin{array}{c}{[-71.6} \\
36.1]\end{array}$ & 35 & 8/28/2019 9:00 & $\begin{array}{c}{[-72.4,} \\
32.5]\end{array}$ \\
\hline al082015 & Tropical Storm Henri & $9 / 9 / 20154: 30$ & $\begin{array}{c}{[-61.5} \\
30.8]\end{array}$ & $\begin{array}{c}9 / 11 / 2015 \\
21: 00\end{array}$ & $\begin{array}{c}{[-58.5,} \\
40.0]\end{array}$ & 35 & 9/11/2015 3:00 & $\begin{array}{c}{[-60.7,} \\
34.8]\end{array}$ \\
\hline al092010 & $\begin{array}{c}\text { Tropical Storm } \\
\text { Gaston }\end{array}$ & $\begin{array}{c}9 / 1 / 2010 \\
15: 00\end{array}$ & $\begin{array}{c}{[-35.8} \\
12.4]\end{array}$ & $\begin{array}{l}9 / 2 / 2010 \\
21: 00\end{array}$ & $\begin{array}{c}{[-39.5} \\
13.5]\end{array}$ & 35 & 9/2/2010 3:00 & $\begin{array}{c}{[-37.7,} \\
13.4]\end{array}$ \\
\hline al092014 & $\begin{array}{c}\text { Tropical Storm } \\
\text { Hanna }\end{array}$ & $\begin{array}{c}10 / 22 / 2014 \\
3: 00\end{array}$ & $\begin{array}{c}{[-92.9} \\
19.4]\end{array}$ & $\begin{array}{c}10 / 28 / 2014 \\
3: 00\end{array}$ & $\begin{array}{c}{[-84.5} \\
14.5]\end{array}$ & 35 & $\begin{array}{c}10 / 27 / 2014 \\
18: 00\end{array}$ & $\begin{array}{c}{[-83.5,} \\
14.9]\end{array}$ \\
\hline al102012 & Tropical Storm Joyce & $\begin{array}{c}8 / 22 / 2012 \\
15: 00\end{array}$ & $\begin{array}{c}{[-36.3} \\
12.4]\end{array}$ & $\begin{array}{c}8 / 24 / 2012 \\
15: 00\end{array}$ & $\begin{array}{c}{[-46.2} \\
17.8]\end{array}$ & 35 & $\begin{array}{c}8 / 23 / 2012 \\
21: 00\end{array}$ & $\begin{array}{c}{[-42.5,} \\
15.9]\end{array}$ \\
\hline
\end{tabular}




\begin{tabular}{|c|c|c|c|c|c|c|c|c|}
\hline al112016 & Tropical Storm Julia & $\begin{array}{c}9 / 14 / 2016 \\
3: 00\end{array}$ & $\begin{array}{c}{[-81.6} \\
30.3]\end{array}$ & $\begin{array}{c}9 / 19 / 2016 \\
3: 00\end{array}$ & $\begin{array}{c}{[-78.4} \\
32.2]\end{array}$ & 35 & $\begin{array}{l}\text { 9/14/2016 } \\
21: 00\end{array}$ & $\begin{array}{c}{[-80.5,} \\
32.1]\end{array}$ \\
\hline al112019 & $\begin{array}{l}\text { Tropical Storm } \\
\text { Imelda }\end{array}$ & $\begin{array}{c}9 / 17 / 2019 \\
17: 00\end{array}$ & $\begin{array}{c}{[-95.4} \\
28.7]\end{array}$ & $\begin{array}{c}9 / 18 / 2019 \\
3: 00\end{array}$ & $\begin{array}{c}{[-95.5} \\
29.8]\end{array}$ & 35 & $\begin{array}{l}9 / 17 / 2019 \\
21: 00\end{array}$ & $\begin{array}{c}{[-95.3,} \\
29.3]\end{array}$ \\
\hline al142008 & Tropical Storm Nana & $\begin{array}{c}10 / 12 / 2008 \\
21: 00\end{array}$ & $\begin{array}{c}{[-37.9} \\
16.4]\end{array}$ & $\begin{array}{c}10 / 14 / 2008 \\
9: 00\end{array}$ & $\begin{array}{c}{[-43.3} \\
18.4]\end{array}$ & 35 & $\begin{array}{c}10 / 13 / 2008 \\
3: 00\end{array}$ & $\begin{array}{c}{[-38.8,} \\
16.6]\end{array}$ \\
\hline al162010 & $\begin{array}{c}\text { Tropical Storm } \\
\text { Nicole }\end{array}$ & $\begin{array}{c}9 / 28 / 2010 \\
15: 00\end{array}$ & $\begin{array}{c}{[-82.5} \\
20.6]\end{array}$ & $\begin{array}{c}9 / 29 / 2010 \\
21: 00\end{array}$ & $\begin{array}{c}{[-80.0} \\
24.5]\end{array}$ & 35 & $\begin{array}{c}9 / 29 / 2010 \\
18: 00\end{array}$ & $\begin{array}{c}{[-80.6,} \\
23.5]\end{array}$ \\
\hline al012009 & $\begin{array}{c}\text { Tropical Depression } \\
\text { One }\end{array}$ & $\begin{array}{c}5 / 28 / 2009 \\
15: 00\end{array}$ & $\begin{array}{c}{[-71.0} \\
37.3]\end{array}$ & $\begin{array}{c}5 / 29 / 2009 \\
21: 00\end{array}$ & $\begin{array}{c}{[-62.3} \\
40.3]\end{array}$ & 30 & 5/29/2009 3:00 & $\begin{array}{c}{[-67.8,} \\
38.1]\end{array}$ \\
\hline al022010 & $\begin{array}{c}\text { Tropical Depression } \\
\text { Two }\end{array}$ & $7 / 8 / 20103: 00$ & $\begin{array}{c}{[-93.9,} \\
23.9]\end{array}$ & $\begin{array}{l}7 / 8 / 2010 \\
21: 00\end{array}$ & $\begin{array}{c}{[-98.4} \\
26.2]\end{array}$ & 30 & 7/8/2010 12:00 & $\begin{array}{c}{[-96.2,} \\
25.7]\end{array}$ \\
\hline al022014 & $\begin{array}{c}\text { Tropical Depression } \\
\text { Two }\end{array}$ & $\begin{array}{c}7 / 21 / 2014 \\
21: 00\end{array}$ & $\begin{array}{c}{[-43.8,} \\
11.6]\end{array}$ & $\begin{array}{c}7 / 23 / 2014 \\
15: 00\end{array}$ & $\begin{array}{c}{[-56.0} \\
14.0]\end{array}$ & 30 & $\begin{array}{c}7 / 22 / 2014 \\
21: 00\end{array}$ & $\begin{array}{c}{[-49.6,} \\
12.9]\end{array}$ \\
\hline al032019 & $\begin{array}{c}\text { Tropical Depression } \\
\text { Three }\end{array}$ & $\begin{array}{c}7 / 22 / 2019 \\
21: 00\end{array}$ & $\begin{array}{c}{[-78.6,} \\
25.6]\end{array}$ & $\begin{array}{c}7 / 23 / 2019 \\
15: 00\end{array}$ & $\begin{array}{c}{[-80.0} \\
29.0]\end{array}$ & 30 & $\begin{array}{c}7 / 23 / 2019 \\
15: 00\end{array}$ & $\begin{array}{r}{[-80.0,} \\
29.0]\end{array}$ \\
\hline al052010 & $\begin{array}{c}\text { Tropical Depression } \\
\text { Five }\end{array}$ & $\begin{array}{c}8 / 10 / 2010 \\
23: 30\end{array}$ & $\begin{array}{c}{[-84.1} \\
26.0]\end{array}$ & $\begin{array}{l}8 / 11 / 2010 \\
21: 00\end{array}$ & $\begin{array}{c}{[-87.6,} \\
28.3]\end{array}$ & 30 & 8/11/2010 6:00 & $\begin{array}{c}{[-84.5,} \\
26.3]\end{array}$ \\
\hline al082009 & $\begin{array}{c}\text { Tropical Depression } \\
\text { Eight }\end{array}$ & $\begin{array}{c}9 / 25 / 2009 \\
21: 00\end{array}$ & $\begin{array}{c}{[-31.6,} \\
16.4]\end{array}$ & $\begin{array}{c}9 / 26 / 2009 \\
21: 00\end{array}$ & $\begin{array}{c}{[-35.7,} \\
18.9]\end{array}$ & 30 & 9/26/2009 9:00 & $\begin{array}{c}{[-33.8,} \\
17.6]\end{array}$ \\
\hline al082013 & $\begin{array}{c}\text { Tropical Depression } \\
\text { Eight }\end{array}$ & $\begin{array}{c}9 / 6 / 2013 \\
18: 30\end{array}$ & $\begin{array}{c}{[-97.8} \\
22.3]\end{array}$ & 9/7/2013 9:00 & $\begin{array}{c}{[-99.3} \\
21.4]\end{array}$ & 30 & 9/6/2013 21:00 & $\begin{array}{c}{[-98.2,} \\
22.2]\end{array}$ \\
\hline al082016 & $\begin{array}{c}\text { Tropical Depression } \\
\text { Eight }\end{array}$ & $\begin{array}{c}8 / 28 / 2016 \\
15: 00\end{array}$ & $\begin{array}{c}{[-70.0} \\
31.5]\end{array}$ & 9/1/2016 9:00 & $\begin{array}{c}{[-69.2} \\
38.2]\end{array}$ & 30 & 8/30/2016 3:00 & $\begin{array}{c}{[-74.1,} \\
33.8]\end{array}$ \\
\hline al092015 & $\begin{array}{c}\text { Tropical Depression } \\
\text { Nine }\end{array}$ & $\begin{array}{c}9 / 16 / 2015 \\
15: 00\end{array}$ & $\begin{array}{c}{[-43.1} \\
15.0]\end{array}$ & $\begin{array}{c}\text { 9/19/2015 } \\
21: 00\end{array}$ & $\begin{array}{c}{[-49.9} \\
18.8]\end{array}$ & 30 & $\begin{array}{c}9 / 17 / 2015 \\
15: 00\end{array}$ & $\begin{array}{c}{[-45.2,} \\
16.1]\end{array}$ \\
\hline al102011 & $\begin{array}{c}\text { Tropical Depression } \\
\text { Ten }\end{array}$ & $\begin{array}{c}8 / 25 / 2011 \\
9: 00\end{array}$ & $\begin{array}{c}{[-30.4,} \\
12.4]\end{array}$ & $\begin{array}{c}8 / 27 / 2011 \\
3: 00\end{array}$ & $\begin{array}{c}{[-34.0,} \\
16.0]\end{array}$ & 30 & $8 / 26 / 20113: 00$ & $\begin{array}{c}{[-33.1} \\
14.1]\end{array}$ \\
\hline al112018 & $\begin{array}{c}\text { Tropical Depression } \\
\text { Eleven }\end{array}$ & $\begin{array}{c}9 / 22 / 2018 \\
3: 00\end{array}$ & $\begin{array}{c}{[-53.4} \\
13.1]\end{array}$ & $\begin{array}{c}9 / 23 / 2018 \\
15: 00\end{array}$ & $\begin{array}{c}{[-56.0} \\
14.5]\end{array}$ & 30 & 9/22/2018 9:00 & $\begin{array}{c}{[-53.5,} \\
13.0]\end{array}$ \\
\hline al152019 & $\begin{array}{c}\text { Tropical Depression } \\
\text { Fifteen }\end{array}$ & $\begin{array}{c}10 / 14 / 2019 \\
21: 00\end{array}$ & $\begin{array}{c}{[-20.2} \\
13.2]\end{array}$ & $\begin{array}{c}10 / 16 / 2019 \\
9: 00\end{array}$ & $\begin{array}{c}{[-24.1} \\
17.3]\end{array}$ & 30 & $\begin{array}{c}10 / 15 / 2019 \\
15: 00\end{array}$ & $\begin{array}{r}{[-21.6,} \\
15.6]\end{array}$ \\
\hline
\end{tabular}




\begin{tabular}{ccccccccc}
\hline \multirow{2}{*}{ al162008 } & Tropical Depression & $10 / 14 / 2008$ & {$[-83.0$,} & $10 / 16 / 2008$ & {$[-85.9$,} & 30 & $10 / 16 / 2008$ & {$[-85.6$,} \\
& Sixteen & $15: 00$ & $15.6]$ & $3: 00$ & $15.3]$ & & $0: 00$ & $15.3]$ \\
& & & & & & & \\
al042017 & Tropical Depression & $7 / 6 / 20173: 00$ & {$[-38.4$,} & $7 / 7 / 2017$ & {$[-52.5$,} & 25 & $7 / 6 / 201721: 00$ & {$[-44.9$,} \\
& & & $12.8]$ & $21: 00$ & $16.5]$ & $13.1]$ \\
\hline
\end{tabular}

Table 2. Excess Twitter activity during storm. Outcome of Twitter data after subtraction of baseline activity levels. Baseline is determined as visualized in Figure 3.

\begin{tabular}{cccccc}
\hline Code & Name & Peak Tweet Date & Peak Tweet Count & Baseline Tweet Level & Total Excess Tweets \\
\hline al052019 & Hurricane Dorian & $2019-09-01$ & 58276 & 1649 & 998729 \\
al132019 & Hurricane Lorenzo & $2019-09-21$ & 6917 & 1124 & 79825 \\
al122019 & Tropical Storm Karen & $2019-09-22$ & 8279 & 2552 & 318994 \\
\hline
\end{tabular}

Table 3. General Linear Model p-values. Output p-values for General Linear Models that determined optimal coefficients for Proximity, Intensity and their Interaction, as explicative variables of excess twitter activity

\begin{tabular}{|c|c|c|c|c|}
\hline Location & Storm & $P>|z|$ for Intensity & $\mathrm{P}>|\mathrm{z}|$ for Proximity & $\mathrm{P}>|\mathrm{z}|$ for Interaction \\
\hline Puerto Rico & Dorian & 0.07619226 & 0.03435301 & 0.05470535 \\
\hline Puerto Rico & Karen & 0.84771792 & 0.90918496 & 0.9538549 \\
\hline Puerto Rico & Lorenzo & 0.3558961853 & - & - \\
\hline Bahamas & Dorian & 0.20105585 & 0.01878344 & 0.05815791 \\
\hline Bahamas & Karen & 0.92925035 & 0.82394846 & 0.9465272 \\
\hline Bahamas & Lorenzo & 0.3558961853 & - & - \\
\hline
\end{tabular}

